\title{
The Interctitical Defocusing Nonlinear Schrödinger Equations with Radial Initial Data in Dimensions Four and Higher
}

\author{
Chuanwei $\mathrm{Gao}^{1}$, Changxing $\mathrm{Miao}^{2, *}$ and Jianwei Yang-Urbain ${ }^{3}$ \\ 1 The Graduate School of China Academy of Engineering Physics, P.O.Box 2101, \\ Beijing 100088, China \\ 2 Institute of Applied Physics and Computational Mathematics, Beijing 100088, China \\ ${ }^{3}$ Department of Mathematics, Beijing Institute of Technology, Beijing 100081, China; \\ and LAGA (UMR CNRS 7539), Université, Paris 13, Sorbonne Paris Cité, \\ Villetaneuse, France
}

Received 2 November 2017; Accepted (in revised version) 20 June 2018

\begin{abstract}
In this paper, we consider the defocusing nonlinear Schrödinger equation in space dimensions $d \geq 4$. We prove that if $u$ is a radial solution which is priori bounded in the critical Sobolev space, that is, $u \in L_{t}^{\infty} \dot{H}_{x}^{s_{c}}$, then $u$ is global and scatters. In practise, we use weighted Strichartz space adapted for our setting which ultimately helps us solve the problems in cases $d \geq 4$ and $0<s_{c}<\frac{1}{2}$. The results in this paper extend the work of [27, Commun. PDEs, 40 (2015), 265-308] to higher dimensions.
\end{abstract}

Key Words: Nonlinear Schrödinger equation, scattering, frequency-localized Morawetz estimae, weighted Strichartz space.

AMS Subject Classifications: 35P25, 35Q55, 47J35

\section{Introduction}

We consider the Cauchy problem for the nonlinear Schrödinger equation (NLS) in $\mathbb{R}_{t} \times \mathbb{R}_{x}^{d}$ with $d \geq 4$ :

$$
\left\{\begin{array}{l}
\left(i \partial_{t}+\Delta\right) u=\mu|u|^{p} u, \\
u(0, x)=u_{0}(x) .
\end{array}\right.
$$

In particular, we call the Eq. (1.1) defocusing when $\mu=1$, and focusing when $\mu=-1$. In this paper, we are dedicated to dealing with the defocusing case.

${ }^{*}$ Corresponding author. Email addresses: canvee@163.com (C. W. Gao), miao_changxing@iapcm.ac.cn (C. X. Miao), jw-urbain.yang@bit.edu.cn, yang@math.univ-paris13.fr (J. W. Yang) 
The solutions of Eq. (1.1) are left invariant by the scaling transformation

$$
u(t, x) \mapsto \lambda^{\frac{2}{p}} u\left(\lambda^{2} t, \lambda x\right)
$$

for $\lambda>0$. This scaling invariance defines a notion of criticality. To be more specified, a direct computation shows that the only homogeneous $L_{x}^{2}$-based Sobolev space that is left invariant by (1.2) is $\dot{H}_{x}^{s_{c}}$, where the critical regularity $s_{c}$ is given by $s_{c}:=\frac{d}{2}-\frac{2}{p}$. We call the problem mass-critical for $s_{c}=0$, energy-critical for $s_{c}=1$ and intercritical for $0<s_{c}<1$. With $s_{c}=\frac{d}{2}-\frac{2}{p}$ in mind, we will transfer from $s_{c}$ to $p$ freely.

We proceed by make the notion of solution precise.

Definition 1.1 (Strong solution). A function $u: I \times \mathbb{R}^{d} \rightarrow \mathbb{C}$ on a non-empty time interval $0 \in I$ is a strong solution to (1.1) if it belongs to $C_{t} \dot{H}_{x}^{s_{c}}\left(K \times \mathbb{R}^{d}\right) \cap L_{t, x}^{\frac{d+2}{2} p}\left(K \times \mathbb{R}^{d}\right)$ for any compact interval $K \subset I$ and obeys the Duhamel formula

$$
u(t)=e^{i t \Delta} u_{0}-i \int_{0}^{t} e^{i(t-s) \Delta}\left(|u|^{p} u\right)(s) d s
$$

for each $t \in I$. We call $I$ the lifespan of $u$. We say that $u$ is a maximal-lifespan solution if it cannot be extended to any strictly larger interval. We say $u$ is a global solution if $I=\mathbb{R}$.

Let $u$ be a maximal-lifespan solution to the problem (1.1), a standard technique shows that the $\|u\|_{L_{t, x}^{\frac{d+2}{2} p}\left(I \times \mathbb{R}^{d}\right)}<\infty$ implies scattering. That is $I=\infty$ and there exists $u_{ \pm} \in \dot{H}_{x}^{s_{c}}\left(\mathbb{R}^{d}\right)$ such that

$$
\lim _{t \rightarrow \pm \infty}\left\|u(t)-e^{i t \Delta} u_{ \pm}\right\|_{\dot{H}_{x}^{s_{c}}\left(\mathbb{R} \times \mathbb{R}^{d}\right)}=0
$$
follows:

The above fact promotes us to define the notion of scattering size and blow up as Definition 1.2 (Scattering size and blow up). We define the scattering size of a solution $u: I \times \mathbb{R}^{d} \rightarrow \mathbb{C}$ to (1.1) by

$$
S_{I}(u):=\iint_{I \times \mathbb{R}^{d}}|u(t, x)|^{\frac{d+2}{2} p} d x d t
$$

If there exists $t_{0} \in I$ so that $S_{\left[t_{0}, \sup I\right)}(u)=\infty$, then we say $u$ blows up forward in time, correspondingly if there exists $t_{0} \in I$ so that $S_{\left(\inf I, t_{0}\right]}(u)=\infty$, then we say $u$ blows up backward in time.

The problem which we concern in this paper can be subsumed into the following conjecture. 
Conjecture 1.1. Let $d \geq 1, p \geq \frac{4}{d}$. Assume $u: I \times \mathbb{R}^{d} \rightarrow C$ is a maximal-lifespan solution to (1.1) such that

$$
u \in L_{t}^{\infty} \dot{H}_{x}^{s_{c}}\left(I \times \mathbb{R}^{d}\right)
$$

then $u$ is global and scatters, with

$$
S_{\mathbb{R}}(u) \leq C\left(\|u\|_{L_{t}^{\infty} \dot{H}_{x}^{s c}}\right)
$$

for some function $C:[0, \infty) \rightarrow[0, \infty)$.

Remark 1.1. When $s_{c}=0$ or $s_{c}=1,(1.4)$ is true as a direct consequence of conservation law. In particular, when $s_{c}=0, u \in L_{t}^{\infty} L_{x}^{2}$ is guaranteed by the mass conservation

$$
M[u(t)]=\int_{\mathbb{R}^{d}}|u(t, x)|^{2} d x .
$$

When $s_{c}=1, u \in L_{t}^{\infty} \dot{H}_{x}^{1}$ follows from the energy conservation

$$
E[u(t)]=\int_{\mathbb{R}^{d}} \frac{1}{2}|\nabla u(t, x)|^{2}+\frac{1}{p+2}|u(t, x)|^{p+2} d x .
$$

For $s_{c} \notin\{0,1\},(1.4)$ can not be deduced from any available conserved quantity and it is a natural artificial assumption as a substitution of conservation law.

Before addressing our main results, we will make a brief review on the Conjecture 1.1. It is well known that in the critical case, the lifespan of solution depends not only on the Sobolev norm but also the profile of the initial data, thus the fact that (1.4) implies the solution $u$ is global and scatters is not at all obvious.

In the energy-critical setting, the breakthrough was made by Bourgain's monumental work [1] in which he introduced the induction on energy method. Based on this method and the space-localized Morawetz inequality, the spherically symmetric energy-critical case was resolved in $d=3,4$. Subsequently, by using the same strategy and the modified interaction Morawetz estimate, Colliander et al. [5] resolved the nonradial case in $d=3$. For further discussion about the defocusing energy-critical NLS, we refer to $[13,20,28$, 34-36]. For focusing case see [10,15,17].

For the mass-critical case, Conjecture 1.1 was primarily proved for sphericallysymmetric $L_{x}^{2}$ initial data in dimensions $d \geq 2$, see [21,31]. By introducing long-time Strichartz estimate method, Dodson in [6-8] settled the nonradial case. The reader may turn to $[9,21,22]$ for focusing setting.

The first work dealing with Conjecture 1.1 at nonconserved critical regularity is attributed to Kenig-Merle [16] at the case $d=3, s_{c}=\frac{1}{2}$ by making use of their pioneered concentration-compactness argument along with Lin-Strauss Morawetz inequality. Note that no additional radial assumption is required in [16] due to the fact that Lin-Strauss Morawetz inequality has a scale of $\frac{1}{2}$. Murphy in [26] extended the result to $d \geq 4$. 
Now we focus on the case $0<s_{c}<\frac{1}{2}$. In [27], under the radial assumption, Murphy handled the case $d=3,0<s_{c}<\frac{1}{2}$ by using long-time Strichartz estimate method and frequencylocalized Lin-Strauss Morawetz estimate. However,it seems not work in higher dimensions, especially $d \geq 5$. To be more precise, following the approach in [27], one can obtain the corresponding result of four dimensions effortlessly. To further generalize that to the higher dimensions, however, is not at all trivial, since it's tricky to establish long-time Strichartz estimate due to the subquadratic property of the nonlinearity. To circumvent the barrier, we exploit the spherical symmetry condition and adopt the strategy of using weighted Strichartz norms as in [31]. The key observation is that one can formulate the weighted Strichartz norm which scales exactly the same as the Strichartz norm of the critical regularity. In doing so, we are liberated from subtle technicality comes from nonlocal nature of the fractional derivative thanks to the fact we place the weight and the derivative at the same height in the sense of scaling which can be exemplified by (3.12a), (3.12b). It's worth mentioning that by adapting the argument in this paper, one may recover the result in [27] for $0<s_{c}<\frac{1}{2}$ in dimension three. We shall clarify this issue at the appropriate point.

For further discussion about Conjecture 1.1, we refer to [11,18, 23, 24].

Now we are in a position to state our main results.

Theorem 1.1. Let $d \geq 4,0<s_{c}<\frac{1}{2}$. Assume that $u: I \times \mathbb{R}^{d}$ is a spherically symmetric maximallifespan solution to (1.1) such that $u \in L_{t}^{\infty} \dot{H}_{x}^{s_{c}}\left(I \times \mathbb{R}^{d}\right)$. Then $u$ is global and scatters, with

$$
S_{\mathbb{R}}(u) \leq C\left(\|u\|_{L_{t}^{\infty} \dot{H}_{x}^{s c}}\right)
$$

for some function $C:[0, \infty) \rightarrow[0, \infty)$.

Adapting the argument in [3], one can obtain the local-in-time theory which serves as a basis for the proof of Theorem 1.1.

Theorem 1.2 (Local Well-posedness). Let $d$ and $s_{c}$ be in the Theorem 1.1, for any $u_{0} \in \dot{H}^{s_{c}}\left(\mathbb{R}^{d}\right)$ and $t_{0} \in \mathbb{R}$, there exists a unique maximal-lifespan solution $u: I \times \mathbb{R}^{d} \rightarrow \mathbb{C}$ to (1.1) with $u\left(t_{0}\right)=u_{0}$. Furthermore

1. Local existence: $I$ is an open neighborhood of $t_{0}$.

2. Blow up: If sup I is finite, then $u$ blows up forward in time. If infI is finite, then $u$ blows up backward in time.

3. Scattering and wave operators: If $\sup I=\infty$ and $u$ does not blow up forward in time, then $u$ scatters forward in time. That is, there exists $u_{+} \in \dot{H}^{s_{c}}\left(\mathbb{R}^{d}\right)$, so that

$$
\lim _{t \rightarrow \infty}\left\|u(t)-e^{i t \Delta} u_{+}\right\|_{\dot{H}^{s c}\left(\mathbb{R}^{d}\right)}=0 .
$$

Conversely, for any $u_{+} \in \dot{H}^{s_{c}}\left(\mathbb{R}^{d}\right)$, there exists a unique solution to (1.1) defined in a neighborhood of $t=\infty$, such that (1.9) holds. The analogous statements hold backward in time. 
4. Small data scattering: If $\left\|u_{0}\right\|_{\dot{H}^{s(}\left(\mathbb{R}^{d}\right)}$ is sufficiently small, then $u$ is global and scatters, with $S_{\mathbb{R}}(u) \lesssim\|u\|_{\dot{H}^{s c}\left(\mathbb{R}^{d}\right)}^{\frac{d+2}{2} p}$

Remark 1.2. To prove Theorem 1.2, one may first assume the initial data belongs to $H_{x}^{s_{c}}$ so that the techniques in [3] applies and then establish Theorem 1.2 by using the following stability lemma.

Lemma 1.1. Let $d \geq 4$, I be a compact interval, and $\tilde{u}: I \times \mathbb{R}^{d} \rightarrow \mathbb{C}$ be a solution to the equation

$$
\left\{\begin{array}{l}
\left(i \partial_{t}+\Delta\right) \tilde{u}=F(\tilde{u})+e \\
\tilde{u}(0)=\tilde{u}_{0} \in \dot{H}_{x}^{s_{c}}
\end{array}\right.
$$

Suppose

$$
\|\tilde{u}\|_{L_{t}^{\infty} \dot{H}_{x}^{s c}\left(I \times \mathbb{R}^{d}\right)} \leq E \quad \text { and } \quad\|\tilde{u}\|_{L_{t, x}{ }^{2}}{ }_{\left(I \times \mathbb{R}^{d}\right)} \leq L,
$$

for some $E, L>0$. There exists $\varepsilon_{1}(E, L)$ such that if $u_{0} \in \dot{H}_{x}^{s_{c}}$ and

$$
\left\|u_{0}-\tilde{u}_{0}\right\|_{\dot{H}_{x}^{s_{c}}}+\left\||\nabla|{ }^{s_{c}} e\right\|_{\mathrm{N}(\mathrm{I})} \leq \varepsilon,
$$

for some small $0<\varepsilon<\varepsilon_{1}(E, L)$, then there exists a solution $u$ to the Eq. (1.1) with the initial data $u_{0}$ and a constant $0<c(d)$ such that

$$
\begin{aligned}
& \left\||\nabla|^{s_{c}}(u-\tilde{u})\right\|_{S(\mathrm{I})} \leq C(E, L) \mathcal{\varepsilon}^{c}, \\
& \left\||\nabla|^{s^{c}} u\right\|_{S(\mathrm{I})} \leq C(E, L),
\end{aligned}
$$

where the definition of $S(I)$ and $N(I)$ can be found in the appendix.

We present the details of the proof of Lemma 1.1 in the Appendix.

Now we can sketch the proof of Theorem 1.1.

\subsection{Reduction to a critical solution}

To prove Theorem 1.1, we argue by contradiction. Due to Theorem 1.2, we know small initial data implies the theory of global existence and scattering. If Theorem 1.1 fails, there exists a counterexample acting as a threshold. As a consequence of its criticality, such counterexample must concentrate in frequency and physical space at the same time. Further analysis shows that such special solution possesses a wealth of weird properties that a solution should not have in general. Finally, we will show that such properties are inconsistent with the structure of the Eq. (1.1).

Definition 1.3. For $A>0$, we define $\mathcal{B}(A)$ as follows

$$
\begin{gathered}
\mathcal{B}(A)=\left\{u_{0} \in \dot{H}_{x}^{s_{c}}, \text { radial }: u: I \times \mathbb{R}^{d}\right. \text { is a maximal-lifespan solution to (1.1) with } \\
\left.u(0)=u_{0} \in \dot{H}_{x}^{s_{c}}, \text { then } \sup _{t \in I}\|u\|_{\dot{H}_{x}^{s_{c}}} \leq A\right\} .
\end{gathered}
$$


Definition 1.4. We say $\mathcal{S C}(A)$ holds if for each $u_{0} \in \mathcal{B}(A)$, then $I=\mathbb{R}$ and $S_{I}(u)<\infty$. Similarly, we say $\mathcal{S C}\left(A, u_{0}\right)$ holds if $u_{0} \in \mathcal{B}(A)$, then $I=\mathbb{R}$ and $S_{I}(u)<\infty$.

In view of (1.4), to prove Theorem 1.1, it suffices to show that $\mathcal{S C}(A)$ holds for each $A>0$. Note that Theorem 1.2 implies $\mathcal{S C}(A)$ holds whenever $A$ is sufficiently small. Consequently, if Proposition 1.1 fails, there exists a critical value $A_{c}$ such that $\operatorname{SC}(A)$ holds when $A<A_{c}$ but fails when $A>A_{c}$. In particular, using concentration-compactness method, we can obtain the following key proposition.

Proposition 1.1. Let $d \geq 4,0<s_{c}<\frac{1}{2}$, if Proposition 1.1 fails, there exists a critical value $A_{c}$ and a critical element $u_{0, c} \in \mathcal{B}\left(A_{c}\right)$ such that $\mathcal{S C}\left(A_{c}, u_{0, c}\right)$ fails. Correspondingly, we call $u_{c}: I \times \mathbb{R}^{d}$ the critical maximal-lifespan solution to (1.1) with $u_{c}(0)=u_{0, c}$.

The derivation of Theorem 1.1 by now is standard. One can refer to $[12,14,18,25-27]$ for more details.

The critical solution $u_{c}$ in Proposition 1.1 enjoys plenty of additional properties, especially among which is its compactness (modulo scaling), see [14,25]. For brevity, in what follows we abbreviate the critical solution $u_{c}$ as $u$.

Proposition 1.2. Let $u: I \times \mathbb{R}^{d}$ be the critical spherically symmetric maximal-lifespan solution to (1.1), for each $\eta>0$, there exists functions $N: I \rightarrow \mathbb{R}^{+}, C: \mathbb{R}^{+} \rightarrow \mathbb{R}^{+}$, such that

$$
\left.\left.\int_{|x| \geq \frac{C(\eta)}{N(t)}}|| \nabla\right|^{s_{c}} \mathcal{u}(t, x)\right|^{2} d x+\int_{|\xi| \geq C(\eta) N(t)}|\xi|^{2 s_{c}}|\hat{u}(t, \xi)|^{2} d \xi<\eta,
$$

for all $t \in I$. We call $N(t)$ the frequency scale function, and $C(\eta)$ the compactness modulus function.

Remark 1.3. 1. This definition is adapted to the radial setting. In the general case, one should also take into account the translation. If we consider mass-critical case, one more parameter should be added in (1.13) due to Galilean invariance of (1.1).

2. By the Arzelà-Ascoli theorem, (1.13) can be rephrased as

$$
\{u(t): t \in I\} \subset\left\{\lambda^{\frac{d-2 s_{c}}{2}} f(\lambda x): \lambda \in(0, \infty) \text { and } f \in K\right\},
$$

where $K$ is a precompact set in $\dot{H}^{s_{c}}$. By $\dot{H}^{s_{c}} \hookrightarrow L_{x}^{\frac{2 d}{d-2 s_{c}}}$, we know that $u$ is also compact (modulo scaling) in $L_{x}^{\frac{2 d}{d-2 s_{c}}}$, that is

$$
\int_{|x| \geq \frac{C(\eta)}{N(t)}}|u(t, x)|^{\frac{2 d}{d-2 s_{c}}} d x \leq \eta
$$

3. We claim that there is a constant $c>0$ such that

$$
\inf _{t \in I}\|u(t)\|_{L_{x}^{d-2 s_{c}}} \geq c .
$$


Otherwise, as $L^{\frac{2 d}{d-2 s_{c}}}$ norm is left invariant under scaling (1.2), there exists a sequence $\left\{N\left(t_{n}\right)^{\frac{-d+2 s_{c}}{2}} u\left(t_{n}, \frac{x}{N\left(t_{n}\right)}\right): t_{n} \in I\right\}$ such that

$$
N\left(t_{n}\right)^{\frac{-d+2 s_{c}}{2}} u\left(t_{n}, \frac{x}{N\left(t_{n}\right)}\right) \rightarrow 0 \quad \text { in } L_{x}^{\frac{2 d}{d-2 s_{c}}}
$$

On the other hand, since $N\left(t_{n}\right)^{\frac{-d+2 s_{c}}{2}} u\left(t_{n}, \frac{x}{N\left(t_{n}\right)}\right)$ is also compact in $\dot{H}_{x}^{s_{c}}$, we have

$$
N\left(t_{n}\right)^{\frac{-d+2 s_{c}}{2}} u\left(t_{n}, \frac{x}{N\left(t_{n}\right)}\right) \rightarrow 0 \text { in } \dot{H}_{x}^{s_{c}}
$$

which contradicts the fact that $u$ blows up.

We emphasize that (1.16) has its analogue in Section 6 of [5] which says the potential part must have lower bound. Further, from the compactness property, we may choose $c(\eta)$ sufficiently small such that

$$
\left.\left.\int_{|x| \leq \frac{c(\eta)}{N(t)}}|| \nabla\right|^{s_{c}} \mathcal{u}(t, x)\right|^{2} d x+\int_{|\xi| \leq c(\eta) N(t)}|\xi|^{2 s_{c}}|\hat{u}(t, \xi)|^{2} d \xi<\eta
$$

Next we will record more properties of the critical solution which will be used in what follows.

Lemma 1.2 (Local Constancy [21]). If $u: I \times \mathbb{R}^{d} \rightarrow \mathbb{C}$ is the critical maximal-lifespan solution to (1.1), then there exists $\delta=\delta(u)>0$ so that for all $t_{0} \in I$

$$
\left[t_{0}-\delta N\left(t_{0}\right)^{-2}, t_{0}+\delta N\left(t_{0}\right)^{-2}\right] \subset I .
$$

Moreover, $N(t) \sim_{u} N\left(t_{0}\right)$ for $\left|t-t_{0}\right| \leq \delta N\left(t_{0}\right)^{-2}$.

Due to Lemma 1.2, we can subdivide the lifespan interval $I$ into several characteristic subintervals $J_{k}$ such that

$$
I=\cup_{k} J_{k}, \quad N(t) \sim N_{k} \quad \text { when } t \in J_{k} \quad \text { with }\left|J_{k}\right| \sim N_{k}^{-2} .
$$

The following result can be directly derived from Lemma 1.2.

Corollary 1.1. Let $u: I \times \mathbb{R}^{d} \rightarrow \mathbb{C}$ be the critical maximal-lifespan solution to (1.1). If $T$ is a finite endpoint of $I$, then $N(t) \gtrsim_{u}|T-t|^{-1 / 2}$. In particular, $\lim _{t \rightarrow T} N(t)=\infty$. lemma.

Finally we relate the frequency function $N(t)$ to spacetime norm by the following

Lemma 1.3 (Spacetime Bound [21]). Let $u: I \times \mathbb{R}^{d} \rightarrow \mathbb{C}$ be the critical maximal-lifespan solution to (1.1), for each interval $J \subset I$, we have

$$
\int_{J} N(t)^{2} d t \lesssim u\left\||\nabla|{ }^{s_{c}} u\right\|_{L_{t}^{2} L_{x}^{\frac{2 d}{d-2}}\left(J \times \mathbb{R}^{d}\right)} \lesssim_{u} 1+\int_{J} N(t)^{2} d t
$$


Remark 1.4. Owing to (1.21), $\int_{I} N(t)^{2} d t$ can be rewritten as follows:

$$
\int_{I} N(t)^{2} d t=\sum_{k} N_{k}^{2}\left|J_{k}\right| \sim \#\left\{J_{k}\right\}
$$

the above formula indicates that the integral of $\int_{I} N(t)^{2} d t$ equals to counting the number of the subintervals $J_{k} \subset I$.

By rescaling argument, we can also ensure

$$
N(t) \leq 1
$$

at least on the interval $J$ which is one direction of maximal lifespan of $u$, say $[0, \sup (I))$. For the sake of exposition, we may harmlessly identify $J$ as $I$. For further discussion, see [31].

To prove Theorem 1.1, it suffices to show that the critical solution in Theorem 1.1 does not exist. To this end, the paper is organized as follows: In Section 2 we will present some basic tools. In Section 3, we will introduce the weighted Strichartz norm and the associated Strichartz estimate. In Section 4, we will establish frequency-localized Morawetz estimate, as a result, we will show that the weighted Strichartz norm of high frequency portion of the solution $u$ will stay bounded, the fact which we will apply directly to rule out the critical solution. In Section 5, we will show that the frequency scale function $N(t)$ can't go to zero. Together with (1.23), ultimately we will preclude the critical solution in Section 6.

\section{Notation and some basic tools}

We write $X \lesssim Y$ or $Y \gtrsim X$ whenever $X \leq C Y$ for some constant $C>0$ and use $\mathcal{O}(Y)$ to denote any quantity $X$ such that $|X| \lesssim Y$. If $X \lesssim Y$ and $Y \lesssim X$ hold simultaneously, we abbreviate that by $X \sim Y$. Without special clarification, the implicit constant $C$ can vary from line to line. We use Japanese bracket $\langle x\rangle$ to denote $\left(1+|x|^{2}\right)^{\frac{1}{2}}$. We denote by $X \pm$ quantity of the form $X \pm \varepsilon$ for any $\varepsilon>0$.

For any spacetime slab $I \times \mathbb{R}^{d}$, we use $L_{t}^{q} L_{x}^{r}\left(I \times \mathbb{R}^{d}\right)$ to denote the Banach space of functions $u: I \times \mathbb{R}^{d} \rightarrow \mathbb{C}$ whose norm is

$$
\|u\|_{L_{t}^{q} L_{x}^{r}\left(I \times \mathbb{R}^{d}\right)}:=\left(\int_{I}\|u(t)\|_{L_{x}^{r}}^{q} d t\right)^{\frac{1}{q}}<\infty,
$$

with the appropriate modification for the case $q$ or $r$ equals to infinity. When $q=r$, for brevity, sometimes we write it as $L_{t, x}^{q}$. One more thing to be noticed is that without obscurity we will use $L_{t}^{q} L_{x}^{r}$ with $L^{q} L^{r}$ interchangeably.

We define the Fourier transform on $\mathbb{R}^{d}$ by

$$
\hat{f}:=(2 \pi)^{-\frac{d}{2}} \int_{\mathbb{R}^{d}} e^{-i x \xi} f(x) d x,
$$


and the homogeneous Sobolev norm as

$$
\|f\|_{\dot{H}^{s}\left(\mathbb{R}^{d}\right)}:=\left\||\nabla|^{s} f\right\|_{L_{x}^{2}\left(\mathbb{R}^{d}\right)},
$$

where

$$
\widehat{|\nabla|^{s} f}(\xi):=|\xi|^{s} \hat{f}(\xi) .
$$

Next we will present the Littlewood-Paley decomposition.

Let $\phi(\xi)$ be a radial bump function supported in the ball $\left\{\xi \in \mathbb{R}^{d}:|\xi| \leq \frac{11}{10}\right\}$ and equals to 1 on the ball $\left\{\xi \in \mathbb{R}^{d}:|\xi| \leq 1\right\}$. For each number $N>0$, we define

$$
\begin{aligned}
& \widehat{P_{\leq N} f}(\xi):=\varphi\left(\frac{\xi}{N}\right) \hat{f}(\xi), \\
& \widehat{P_{>N} f}(\xi):=\left(1-\varphi\left(\frac{\xi}{N}\right)\right) \hat{f}(\xi), \\
& \widehat{P_{N} f}(\xi):=\left(\varphi\left(\frac{\xi}{N}\right)-\varphi\left(\frac{2 \xi}{N}\right)\right) \hat{f}(\xi),
\end{aligned}
$$

with similar definitions for $P_{<N}$ and $P_{\geq N}$. Moreover, we define

$$
P_{M<\cdot \leq N}:=P_{\leq N}-P_{\leq M},
$$

whenever $M<N$. Also there are the following Bernstein inequalities for the LittlewoodPaley operators:

$$
\left\{\begin{array}{l}
\left\||\nabla|^{s} P_{\leq N} f\right\|_{L^{q}} \lesssim N^{s}\left\|P_{\leq N} f\right\|_{L^{q}} \lesssim N^{s}\|f\|_{L^{q}} \\
\left\|P_{>N} f\right\|_{L^{q}} \lesssim N^{-s}\left\||\nabla|^{s} P_{>N} f\right\|_{L^{q}} \lesssim N^{-s}\left\||\nabla|^{s} f\right\|_{L^{q}} \\
\left\||\nabla|^{ \pm s} P_{N} f\right\|_{L^{q}} \lesssim N^{ \pm s}\left\|P_{N} f\right\|_{L^{q}} \lesssim N^{ \pm s}\|f\|_{L^{q}} \\
\left\|P_{\leq N} f\right\|_{L^{q}} \lesssim N^{d\left(\frac{1}{p}-\frac{1}{q}\right)}\left\|P_{\leq N} f\right\|_{L^{p}} \\
\left\|P_{N} f\right\|_{L^{q}} \lesssim N^{d\left(\frac{1}{p}-\frac{1}{q}\right)}\left\|P_{\leq N} f\right\|_{L^{p}}
\end{array}\right.
$$

where $1 \leq p \leq q \leq \infty$.

Lemma 2.1 (Fractional product rule [3]). Let $s>0$ and $1<r, r_{j}, q_{j}<\infty$ satisfy $\frac{1}{r}=\frac{1}{r_{j}}+\frac{1}{q_{j}}$ for $j=1,2$, then

$$
\left\||\nabla|^{s}(f g)\right\|_{L_{x}^{r}} \lesssim\|f\|_{L_{x}^{r_{1}}}\left\||\nabla|^{s} g\right\|_{L_{x}^{q_{1}}}+\left\||\nabla|^{s} f\right\|_{L_{x}^{r_{2}}}\|g\|_{L_{x}^{q_{2}}}
$$

We will also need the following chain rule for fractional order derivatives. One can turn to [3] for more details.

Lemma 2.2 (Fractional chain rule). Suppose $G \in C^{1}(\mathbb{C})$ and $s \in(0,1]$. Let $1<r<r_{2}<\infty$ and $1<r_{1} \leq \infty$ be such that $\frac{1}{r}=\frac{1}{r_{1}}+\frac{1}{r_{2}}$, then

$$
\left\||\nabla|^{s} G(u)\right\|_{L_{x}^{r}} \lesssim\left\|G^{\prime}(u)\right\|_{L_{x}^{r_{1}}}\left\||\nabla|^{s} u\right\|_{L_{x}^{r_{2}}} .
$$


When the function $G$ is no longer $\mathbb{C}^{1}$, but merely Hölder continuous, we have the following chain rule:

Lemma 2.3 (Fractional chain rule for Hölder continuous function [35]). Let G be a Hölder continuous function of order $0<\alpha<1$. Then for every $0<s<\alpha, 1<p<\infty$, and $\frac{s}{\alpha}<\sigma<1$, we have

$$
\left\||\nabla|^{s} G(u)\right\|_{p} \lesssim\left\||u|^{\alpha-\frac{s}{\sigma}}\right\|_{p_{1}}\left\||\nabla|^{\sigma} u\right\|_{\frac{s}{\sigma}}^{\frac{s}{\sigma}} p_{2}
$$

provided $\frac{1}{p}=\frac{1}{p_{1}}+\frac{1}{p_{2}}$ and $\left(1-\frac{s}{\alpha \sigma}\right) p_{1}>1$.

The classical Hörmander-Mikhlin theorem concerns about the sufficient condition required for a function to be an $L^{p}(1<p<\infty)$ multiplier. We should adapt the usual one to be suited for our case and present here the extension form with the power weights. One can refer to [29] for further discussion.

Lemma 2.4. Let $T$ be a Hörmander-Mikhlin multiplier defined on tempered function $f$ i.e.,

$$
\widehat{T f}(\xi):=m(\xi) \hat{f}(\xi),
$$

with its symbol $m(\xi)$ satisfying the following pointwise estimate

$$
\left|\nabla^{\alpha} m(\xi)\right| \lesssim \alpha|\xi|^{-|\alpha|}
$$

for every nonnegative multi-index $\alpha$. Then for any $1<p<\infty$, and $-\frac{d}{p}<s<d-\frac{d}{p}$, we have

$$
\left\||x|^{s} T f\right\|_{L_{x}^{p} \lesssim p, s}\left\||x|^{s} f\right\|_{L_{x}^{p}}
$$

for all $f$ such that right-hide side is finite.

Remark 2.1. In particular, the operator $N^{-s}|\nabla|{ }^{s} P_{<N}$ and $N^{s}|\nabla|^{-s} P_{\geq N}$ are all HörmanderMikhlin multiplier, as well as the frequency localized operator $P_{N}, P_{\gtrless N}$.

At the end of this section, we will record some fundamental tools. One can find details in [31] and the materials therein.

Lemma 2.5 (Hardy-Littlewood-Sobolev Inequality). Let $1<p, q<\infty, d \geq 1,0<s<d$, and $\alpha, \beta \in \mathbb{R}$ obey the condition

$$
\begin{aligned}
& \alpha>-\frac{d}{p^{\prime}}, \\
& \beta>-\frac{d}{q^{\prime}}, \\
& 1 \leq \frac{1}{p}+\frac{1}{q} \leq 1+s,
\end{aligned}
$$


and the scaling condition

$$
\alpha+\beta-d+s=-\frac{d}{p^{\prime}}-\frac{d}{q^{\prime}} .
$$

Then for any spherically symmetric $u: \mathbb{R}^{d} \rightarrow \mathbb{C}$, we have

$$
\left\||x|^{\beta} u\right\|_{L^{q^{\prime}\left(\mathbb{R}^{d}\right)}} \lesssim \alpha, \beta, p, q,\left.s|| x\right|^{-\alpha}|\nabla|^{s} u \|_{L^{p}\left(\mathbb{R}^{d}\right)} .
$$

Lemma 2.6. If $f: \mathbb{R}^{d} \rightarrow \mathbb{C}, 1<p<\infty, 0<\alpha<\frac{d}{p}$, and $N>0$, then

$$
\left\||x|^{-\alpha} P_{<N} f\right\|_{L^{p}\left(\mathbb{R}^{d}\right)} \lesssim \alpha, p\langle N\rangle^{\alpha}\left\|\langle x\rangle^{-\alpha} f\right\|_{L^{p}\left(\mathbb{R}^{d}\right)} .
$$

\section{Weighted Strichartz inequality}

Motivated by the work of [31] which handled the mass-critical case, we adapt the argument to tackle the case without conserved quantities. In practice, we introduce weighted Strichartz norm suited for our case. To be more precise, we define $\|u\|_{\mathcal{S}\left(I \times \mathbb{R}^{d}\right)}$ and $\|u\|_{\mathcal{N}\left(I \times \mathbb{R}^{d}\right)}$ respectively as follows:

$$
\begin{aligned}
& \|u\|_{\mathcal{S}\left(I \times \mathbb{R}^{d}\right)}=\left\||x|^{-\frac{1+\varepsilon}{2}}|\nabla|^{\frac{1-\varepsilon}{2}+s_{c}} u\right\|_{L_{t, x}^{2}\left(I \times \mathbb{R}^{d}\right)}+\left\||\nabla|^{s_{c}} u\right\|_{L_{t}^{\infty} L_{x}^{2}\left(I \times \mathbb{R}^{d}\right)}, \\
& \|u\|_{\mathcal{N}\left(I \times \mathbb{R}^{d}\right)}=\left\||x|^{\frac{1+\varepsilon}{2}}|\nabla|^{-\frac{1-\varepsilon}{2}+s_{c}} u\right\|_{L_{t, x}^{2}\left(I \times \mathbb{R}^{d}\right)^{\prime}}
\end{aligned}
$$

where $\varepsilon>0$ is a sufficiently small constant depending on $d$ and $s_{c}$. By Lemma 2.4, we obtain that corresponding Bernstein inequalities with respect to the norms $\|u\|_{\mathcal{S}\left(I \times \mathbb{R}^{d}\right)}$ and $\|u\|_{\mathcal{N}\left(I \times \mathbb{R}^{d}\right)}$.

Lemma 3.1. For any $s>0$ and dyadic number $N>0$, we have

$$
\begin{aligned}
& \left\||\nabla|^{s} u_{<N}\right\|_{\mathcal{S}\left(I \times \mathbb{R}^{d}\right)} \lesssim N^{s}\left\|u_{<N}\right\|_{\mathcal{S}\left(I \times \mathbb{R}^{d}\right)^{\prime}} \\
& \left\||\nabla|^{-s} u_{>N}\right\|_{\mathcal{S}\left(I \times \mathbb{R}^{d}\right)} \lesssim N^{-s}\left\|u_{>N}\right\|_{\mathcal{S}\left(I \times \mathbb{R}^{d}\right)^{\prime}} \\
& \left\||\nabla|^{s} u_{<N}\right\|_{\mathcal{N}\left(I \times \mathbb{R}^{d}\right)} \lesssim N^{s}\left\|u_{<N}\right\|_{\mathcal{N}\left(I \times \mathbb{R}^{d}\right)^{\prime}} \\
& \left\||\nabla|^{-s} u_{>N}\right\|_{\mathcal{N}\left(I \times \mathbb{R}^{d}\right)} \lesssim N^{-s}\left\|u_{>N}\right\|_{\mathcal{N}\left(I \times \mathbb{R}^{d}\right)}
\end{aligned}
$$

The association of $\|u\|_{\mathcal{S}\left(I \times \mathbb{R}^{d}\right)}$ and $\|u\|_{\mathcal{N}\left(I \times \mathbb{R}^{d}\right)}$ with Eq. (1.1) is illuminated by the following weighted Strichartz estimate and radial Sobolev embedding.

Proposition 3.1 (Weighted Strichartz estimate [33]). Let $u, G: I \times \mathbb{R}^{d} \rightarrow \mathbb{C}$ satisfy $\left(i \partial_{t}+\Delta\right) u=$ $G$ in the sense of distributions, then we have

$$
\|u\|_{\mathcal{S}\left(I \times \mathbb{R}^{d}\right)} \lesssim\left\|u\left(t_{0}\right)\right\|_{\dot{H}^{s c}\left(\mathbb{R}^{d}\right)}+\|G\|_{\mathcal{N}\left(I \times \mathbb{R}^{d}\right)},
$$

for all $t_{0} \in I$. 
Using (2.5), we will get the following radial Sobolev embedding.

Lemma 3.2 (Radial Sobolev embedding). Let $u$ be spherically symmetric and $d \geq 4$, then we have

$$
\left\||\nabla|^{s_{c}} \mathcal{u}\right\|_{L_{t}^{2} L_{x}^{\frac{2 d}{d-2}}} \lesssim\|u\|_{\mathcal{S}}
$$

Lemma 3.3. If $u, v: I \times \mathbb{R}^{d} \rightarrow \mathbb{C}$ are spherically symmetric and $d \geq 4$, then

$$
\left\||u|^{\frac{4}{d-2 s_{c}}} \mathcal{v}\right\|_{\mathcal{N}} \lesssim\left\||\nabla|^{s_{c}} \mathcal{u}\right\|_{L_{t}^{\infty} L_{x}^{2}}^{\frac{4}{d-2 s_{c}}}\left\||\nabla|^{s_{c}} \mathcal{v}\right\|_{L_{t}^{2} L_{x}^{\frac{2 d}{d-2}}} \lesssim\left\||\nabla|^{s_{c}} u\right\|_{L_{t}^{\infty} L_{x}^{2}}^{\frac{4}{d-2 s_{c}}}\|v\|_{\mathcal{S}}
$$

Proof. Case I: $d=4, p=\frac{2}{2-s_{c}}>1$

By (2.5), we obtain

$$
\left\||x|^{\frac{1+\varepsilon}{2}} u\right\|_{L_{t, x}^{2}} \lesssim\left\||\nabla|^{\frac{1-\varepsilon}{2}} u\right\|_{L_{t}^{2} L_{x}^{\frac{4}{3}}}
$$

By the definition of $\mathcal{N}$, (3.5) implies

$$
\left\||u|^{\frac{2}{2-s_{c}}} v\right\|_{\mathcal{N}} \lesssim\left\||\nabla|^{s_{c}}\left(|u|^{\frac{2}{2-s_{c}}} \mathcal{V}\right)\right\|_{L_{t}^{2} L_{x}^{\frac{4}{3}}} .
$$

Continuing from (3.6), by Lemma 2.1, Lemma 2.2 and (3.3) we have

$$
\begin{aligned}
\text { RHS of(3.6) } & \lesssim\left\||\nabla|^{s_{c}}|u|^{\frac{2}{2-s_{c}}}\right\|{ }_{L_{t}^{\infty} L_{x}^{2+s_{c}}}\|v\|_{L_{t}^{2} L_{x}^{\frac{4}{1-s_{c}}}}+\left\||u|^{\frac{2}{2-s_{c}}}\right\|_{L_{t}^{\infty} L_{x}^{2}}\left\||\nabla|^{s_{c}} v\right\|_{L_{t}^{2} L_{x}^{4}} \\
& \lesssim\left\||\nabla|^{s_{c}} u\right\|_{L_{t}^{\infty} L_{x}^{2}}^{\frac{2}{2-s_{c}}}\left\||\nabla|^{s_{c}} v\right\|_{L_{t}^{2} L_{x}^{4}} \\
& \lesssim\left\||\nabla|^{s_{c}} u\right\|_{L_{t}^{\infty} L_{x}^{2}}^{\frac{2}{2-s_{c}}}\|v\|_{\mathcal{S}}
\end{aligned}
$$

Case II: $d \geq 5, p=\frac{4}{d-2 s_{c}}<1$

If $0<s_{c}<\frac{d-2}{2(d-1)}$, by the definition of $\mathcal{N}$, (2.5) implies

$$
\left\||u|^{\frac{4}{d-2 s_{c}}} \mathcal{v}\right\|_{\mathcal{N}} \lesssim\left\||u|^{\frac{4}{d-2 s_{c}}} \mathcal{V}\right\|_{L_{t}^{2} L_{x}^{\frac{2 d}{d+2-2 s_{c}}}}
$$

Continuing from (3.7), by the Hölder inequality and (3.3) we have

$$
\begin{aligned}
& \lesssim\|u\|^{\frac{4}{d-2 s_{c}}}\|v\|_{L_{t}^{\infty} L_{x}^{\frac{2 d}{d-2 s_{c}}}} \frac{2 d}{L_{t}^{2} L_{x}^{\frac{2-2\left(1+s_{c}\right)}{d}}} \\
& \lesssim\left\||\nabla|^{s_{c}} u\right\|_{L_{t}^{\infty} L_{x}^{2}}^{\frac{4}{d-2 S_{c}}}\|v\|_{\mathcal{S}} .
\end{aligned}
$$


When $\frac{d-2}{2(d-1)} \leq s_{c}<\frac{1}{2}$, denoting $\bar{s}=\left(s_{c}-\frac{d-2}{2(d-1)}\right)+$, similarly by (2.5) we have

$$
\left\||u|^{\frac{4}{d-2 s_{c}}} v\right\|_{\mathcal{N}} \lesssim\left\||\nabla|^{\bar{s}}\left(|u|^{\frac{4}{d-2 s_{c}}} v\right)\right\|_{L_{t}^{2} L_{x}^{\frac{2 d}{d+2-2\left(s_{c}-\bar{s}\right)}}} .
$$

Continuing from (3.8), by Lemma 2.1 we have

$$
\begin{aligned}
& \text { RHS of (3.8) } \\
& \lesssim\left\||\nabla|^{\bar{s}}|u|^{\frac{4}{d-2 s_{c}}}\right\|{ }_{L_{t}^{\infty} L_{x}^{\frac{d}{2+\bar{s}}}}\|v\|_{L_{t}^{2} L_{x}^{\frac{2 d}{d-2-2 s_{c}}}}+\left\||u|^{\frac{4}{d-2 s_{c}}}\right\|_{L_{t}^{\infty} L_{x}^{\frac{d}{2}}}\left\||\nabla|^{\bar{s}} v\right\|_{L_{t}^{2} L_{x}^{\frac{1}{d-2-2\left(s_{c}-\bar{s}\right)}}} \\
& \lesssim\left\||\nabla|^{\bar{s}}|u|^{\frac{4}{d-2 s_{c}}}\right\|_{L_{t}^{\infty} L_{x}^{\frac{d}{2+\bar{s}}}}\left\||\nabla|^{s_{c}} v\right\|_{L_{t}^{2} L_{x}^{\frac{2 d}{d-2}}}+\left\||\nabla|^{s_{c}} u\right\|_{L_{t}^{\infty} L_{x}^{2}}^{\frac{4}{d-s_{c}}}\left\||\nabla|^{s_{c}} v\right\|_{L_{t}^{2} L_{x}^{\frac{2 d}{d-2}}} .
\end{aligned}
$$

To complete the proof, it suffices to show that

$$
\left\||\nabla|^{\bar{s}}|u|^{\frac{4}{d-2 s_{c}}}\right\|_{L_{t}^{\infty} L_{x}^{\frac{d}{2+\bar{s}}}} \lesssim\left\||\nabla|^{s_{c}} \mathcal{u}\right\|_{L_{t}^{\infty} L_{x}^{2}}^{\frac{4}{d-2 s_{c}}}
$$

To this end, setting $\sigma=\frac{\bar{s}}{p}+\tilde{\varepsilon}$, where $\tilde{\varepsilon}$ is a sufficiently small positive constant (say, $\tilde{\varepsilon}=\frac{1}{2^{10}}$ ). Using Lemma 2.3 with $\alpha$ being replaced by $\frac{4}{d-2 s_{c}}$, we have

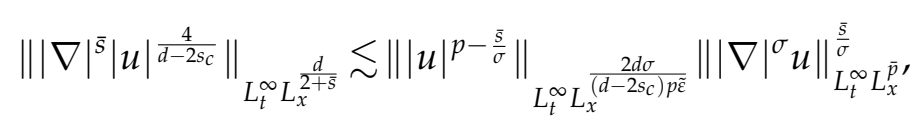

where $\bar{p}=\frac{2 d \bar{s}}{2(2+\bar{s}) \sigma-\left(d-2 s_{c}\right) \overline{\tilde{\varepsilon}}}$, using Sobolev inequality we have (3.9).

By the local well-posed theory, for example see [2], one has

$$
\left\||\nabla|^{s_{c}} u\right\|_{L_{t}^{2} L_{x}^{\frac{2 d}{d-2}}\left(J \times \mathbb{R}^{d}\right)}<\infty
$$

for any compact interval $J$ contained in the maximal lifespan interval $I$. As a direct application of (3.4), we obtain the following result which, in some sense, can be viewed as an extension of (3.11) in the weighted norm.

Corollary 3.1. Let $u: I \times \mathbb{R}^{d} \rightarrow \mathbb{C}$ be a spherically symmetric maximal-lifespan solution to (1.1) then

$$
\|u\|_{\mathcal{S}\left(J \times \mathbb{R}^{d}\right)}<\infty \quad \text { for all compact set } J \subset \subset I .
$$

Proof. Using (1.4), (3.2) (3.4) and (3.11), we obtain

$$
\begin{aligned}
\|u\|_{\mathcal{S}\left(J \times \mathbb{R}^{d}\right)} & \lesssim 1+\left\||u|^{\frac{4}{d-2 s_{c}}} u\right\|_{\mathcal{N}} \\
& \lesssim\left\||\nabla|^{s^{c}} u\right\|_{L_{t}^{\infty} L_{x}^{2}}^{\frac{4}{d-2 s}}\left\||\nabla|^{s_{c}} u\right\|_{L_{t}^{2} L_{x}^{\frac{2 d}{d-2}}} \\
& <\infty
\end{aligned}
$$

Thus, we complete the proof. 
Next, we will give some refined nonlinear estimates which will be used to control the nonlinear interaction.

Proposition 3.2 (Refined nonlinear estimate). Let $u, v: I \times \mathbb{R}^{d} \rightarrow \mathbb{C}$ be spherically symmetric, then we have

$$
\begin{aligned}
& \left\||\nabla|^{\frac{1-\varepsilon}{2}-s_{c}} \mathcal{O}\left(|u|^{\frac{4}{d-2 s_{c}}} v\right)\right\|_{\mathcal{N}} \lesssim\left\||\nabla|^{\left(\frac{d}{2}-\frac{\left(d-2 s_{c}\right)\left(1+s_{c}+\varepsilon\right)}{4}\right)-} u\right\|_{L_{t}^{\infty} L_{x}^{2}}^{\frac{4}{d-2 s_{c}}}\left\||\nabla|^{\left(-\frac{1-\varepsilon}{2}\right)+} v\right\|_{\mathcal{S}} \\
& \left\||\nabla|^{\frac{1-\varepsilon}{2}-s_{c}} \mathcal{O}\left(|u|^{\frac{4}{d-2 s_{c}}} v\right)\right\|_{\mathcal{N}} \lesssim\left\||\nabla|^{\frac{1+\varepsilon}{2}} u\right\|_{L_{t}^{\infty} L_{x}^{2}}^{\frac{4}{d-2 s_{c}}}\left\||\nabla|^{\frac{1-\varepsilon}{2}-s_{c}+p\left(s_{c}-\frac{1+\varepsilon}{2}\right)} v\right\|_{\mathcal{S}}
\end{aligned}
$$

Proof. By the definition of $\mathcal{N}$ and the Hölder inequality and Lemma 2.5, we estimate (3.12a) as

$$
\begin{aligned}
& \left\||\nabla|^{\frac{1-\varepsilon}{2}-s_{c}} \mathcal{O}\left(|u|^{\frac{4}{d-2 s_{c}}} v\right)\right\|_{\mathcal{N}} \\
\lesssim & \left\||x|^{\frac{1+\varepsilon}{2}} \mathcal{O}\left(|u|^{\frac{4}{d-2 s_{c}}} v\right)\right\|_{L_{t, x}^{2}} \\
\lesssim & \left\||x|^{1+\varepsilon+s_{c}}|u|^{\frac{4}{d-2 s_{c}}}\right\|_{L_{t}^{\infty} L_{x}^{\infty-}}^{\infty}\left\||x|^{-\frac{1+\varepsilon}{2}-s_{c}} \mathcal{V}\right\|_{L_{t}^{2} L_{x}^{2+}} \\
\lesssim & \left\||\nabla|^{\left(\frac{d}{2}-\frac{\left(d-2 s_{c}\right)\left(1+s_{c}+\varepsilon\right)}{4}\right)-} u\right\|_{L_{t}^{\infty} L_{x}^{2}}^{\frac{4}{d-2 s_{c}}}\left\||\nabla|^{\left(-\frac{1-\varepsilon}{2}\right)+} v\right\|_{\mathcal{S}} .
\end{aligned}
$$

Similarly for (3.12b), we have

$$
\begin{aligned}
& \left\||\nabla|^{\frac{1-\varepsilon}{2}-s_{c}} \mathcal{O}\left(|u|^{\frac{4}{d-2 s_{c}}} v\right)\right\|_{\mathcal{N}} \\
\lesssim & \left\||x|^{\frac{1+\varepsilon}{2}} \mathcal{O}\left(|u|^{\frac{4}{d-2 s_{c}}} v\right)\right\|_{L_{t, x}^{2}} \\
\lesssim & \|u\|^{\frac{4}{d-2 s_{c}}} \frac{2 d}{L_{t}^{\infty} L_{x}^{(d-1-\varepsilon))}}\left\||x|^{\frac{1+\varepsilon}{2}} v\right\|_{L_{t}^{2} L_{x}^{\frac{2 d}{d-(d-1-\varepsilon) p}}} \\
\lesssim & \left\||\nabla|^{\frac{1+\varepsilon}{2}} u\right\|_{L_{t}^{\infty} L_{x}^{2}}^{\frac{4}{d-2 s_{c}}}\left\||\nabla|^{\frac{1-\varepsilon}{2}-s_{c}+p\left(s_{c}-\frac{1+\varepsilon}{2}\right)} v\right\|_{\mathcal{S}} .
\end{aligned}
$$

Thus, we complete the proof.

Remark 3.1. (3.12a) is very useful when $u$ is low frequency and $v$ is high frequency, as it transfers plenty of derivatives from high frequency to low frequency via the appropriate distribution of weight.

\section{Frequency-localized Morawetz estimate}

In this part we will primarily establish the following frequency-localized Morawetz inequality. 
Proposition 4.1 (Frequency-localized Morawetz estimate). Let $d \geq 4$ and $u: I \times \mathbb{R}^{d} \rightarrow \mathbb{C}$ be the critical spherically symmetric maximal-lifespan solution to (1.1) which obeys (1.4), (1.23), then we have

$$
\lim _{N \rightarrow \infty} N^{2 s_{c}} \int_{I} \int_{\mathbb{R}^{d}} \frac{\left|\nabla u_{<N}(t, x)\right|^{2}}{|N x|^{1+\varepsilon}} d x d t=0 .
$$

To prove Proposition 4.1, we will first exploit some nontrivial facts about the critical solution $u$.

Lemma 4.1. Let $u: I \times \mathbb{R}^{d} \rightarrow \mathbb{C}$ be the critical spherically symmetric maximal-lifespan solution to (1.1) which obeys (1.4), (1.23). Then for each $\theta>0$, we have

$$
\lim _{N \rightarrow \infty}\left(\left\||\nabla|^{s_{c}} \mathcal{u}_{\geq N}\right\|_{L_{t}^{\infty} L_{x}^{2}\left(I \times \mathbb{R}^{d}\right)}+\frac{1}{N^{\theta}}\left\||\nabla|^{\theta+s_{c}} \mathcal{u}_{<N}\right\|_{L_{t}^{\infty} L_{x}^{2}\left(I \times \mathbb{R}^{d}\right)}\right)=0 .
$$

Proof. By (1.13) and (1.23), we have that

$$
\lim _{N \rightarrow \infty}\left\||\nabla|^{s_{c}} \mathcal{u}_{>N}\right\|_{L_{t}^{\infty} L_{x}^{2}}=0
$$

Now we turn to proving the second term, we split $u_{<N}$ as $u_{<N}:=u_{<\sqrt{N}}+u_{\sqrt{N} \leq .<N}$ then by Bernstein inequality, we have

$$
\begin{aligned}
& \frac{1}{N^{\theta}}\left\||\nabla|^{\theta+s_{c}} u_{<N}\right\|_{L_{t}^{\infty} L_{x}^{2}} \\
\lesssim & \frac{1}{N^{\theta}}\left\||\nabla|^{\theta+s_{c}} u_{<\sqrt{N}}\right\|_{L_{t}^{\infty} L_{x}^{2}}+\frac{1}{N^{\theta}}\left\||\nabla|^{\theta+s_{c}} u_{\sqrt{N} \leq .<N}\right\|_{L_{t}^{\infty} L_{x}^{2}} \\
\lesssim & \frac{1}{N^{\frac{\theta}{2}}}\left\||\nabla|^{s_{c}} u_{<\sqrt{N}}\right\|_{L_{t}^{\infty} L_{x}^{2}}+\left\||\nabla|^{s_{c}} u_{\sqrt{N} \leq .<N}\right\|_{L_{t}^{\infty} L_{x}^{2}} \\
\rightarrow & 0 \text { as } N \rightarrow \infty .
\end{aligned}
$$

Thus, we complete the proof of the lemma.

In view of this Lemma 4.1, we can reformulate Proposition 4.1 as follows

Theorem 4.1 (Frequency-localized Morawetz estimate I). Let $d \geq 4,0<\eta<1$, and $u: I \times \mathbb{R}^{d}$ be the critical spherically symmetric maximal-lifespan solution to (1.1) which satisfies (1.4), (1.23). Then there exits $\delta>0$ with the following property: given any $N>0$ such that

$$
\left\||\nabla|^{s_{c}} \mathcal{u}_{\geq N}\right\|_{L_{t}^{\infty} L_{x}^{2}\left(I \times \mathbb{R}^{d}\right)}+\frac{1}{N^{\theta}}\left\||\nabla|^{\theta+s_{c}} \mathcal{u}_{<N}\right\|_{L_{t}^{\infty} L_{x}^{2}\left(I \times \mathbb{R}^{d}\right)} \leq \delta,
$$

we have

$$
N^{2 s_{c}} \int_{I} \int_{\mathbb{R}^{d}} \frac{\left|\nabla u_{<N}(t, x)\right|^{2}}{|N x|^{1+\varepsilon}} d x d t \leq \eta .
$$


By scaling invariance of the Eq. (1.1), we may choose $N=1$. By a limiting argument, we may then take $I$ to be compact. Indeed, observe that by Corollary 3.1, the left-hand side of (4.4) varies continuously on $I$ and goes to zero when I shrinks to a point. Thus, by standard continuity argument, it suffices to show the following bootstrap version of Proposition 4.1.

Proposition 4.2 (Frequency-localized Morawetz estimate II). Let $d \geq 4,0<\eta<1$, and $u: I \times \mathbb{R}^{d}$ be the critical symmetric solution to (1.1) which satisfies (1.4), (1.23). Then there exits $\delta>0$ with the following property:

$$
\left\||\nabla|^{s_{c}} u_{h i}\right\|_{L_{t}^{\infty} L_{x}^{2}\left(I \times \mathbb{R}^{d}\right)}+\left\||\nabla|^{\theta+s_{c}} u_{l o}\right\|_{L_{t}^{\infty} L_{x}^{2}\left(I \times \mathbb{R}^{d}\right)} \leq \delta,
$$

where $u_{h i}:=u_{\geq 1}$ and $u_{l o}:=u_{<1}$, such that we also have bootstrap hypothesis: if

$$
Q_{I}:=\int_{I} \int_{\mathbb{R}^{d}} \frac{\left|\nabla u_{l o}(t, x)\right|^{2}}{|x|^{1+\varepsilon}} d x d t \leq 2 \eta
$$

then we have

$$
Q_{I} \leq \eta
$$

In order to prove Proposition 4.2, we will primarily establish the corresponding estimate for low and high frequency portion of the solution $u$.

Lemma 4.2 (Low and high frequency bound). Under the conditions of Proposition 4.2, we have the following estimates:

$$
\begin{aligned}
& \left\||\nabla|^{\frac{1+\varepsilon}{2}} u_{l o}\right\|_{\delta\left(I \times \mathbb{R}^{d}\right)} \lesssim \eta^{1 / 2}, \\
& \left\|\nabla u_{l o}\right\|_{L_{t}^{2} L_{x}^{\frac{2 d}{d-2\left(1-\varepsilon_{0}\right)}}\left(I \times \mathbb{R}^{d}\right)} \lesssim \eta^{1 / 2}, \\
& \left\|u_{h i}\right\|_{\delta\left(I \times \mathbb{R}^{d}\right)} \lesssim \delta+\delta \delta^{\frac{4}{d-2 s_{c}}}
\end{aligned}
$$

where $\varepsilon_{0}(d)>0$ is sufficiently small.

Proof. From the definition of $\mathcal{S}$, Lemmas 3.1 (4.5) and (4.6) we derive (4.7a) by choosing $\delta$ sufficiently small. (4.7b) comes from (4.7a) and (2.5). Indeed, by Lemma 3.1 and choosing $\varepsilon_{0}$ sufficiently small, we have

$$
\left\|\nabla u_{l o}\right\|_{L_{t}^{2} L_{x}^{\frac{2 d}{d-2\left(1-\varepsilon_{0}\right)}}} \lesssim\left\||\nabla|^{\frac{1+\varepsilon}{2}+s_{c}+\varepsilon_{0}} u_{l o}\right\|_{L_{t}^{2} L_{x}^{\frac{2 d}{d-2\left(1-\varepsilon_{0}\right)}}} .
$$

By (4.7a) and (2.5), we get (4.7b).

Now it suffices to prove (4.7c). We denote $P_{h i}:=P_{\geq 1}$. Obviously

$$
\left(i \partial_{t}+\Delta\right) P_{h i} u=P_{h i} F(u) .
$$


By Strichartz estimate (3.2), (4.5) and splitting $P_{h i} F(u)$ into

$$
P_{h i} F(u)=P_{h i} F\left(u_{l o}\right)+P_{h i}\left(F(u)-F\left(u_{l o}\right)\right),
$$

we have

$$
\begin{aligned}
\left\|u_{h i}\right\|_{\mathcal{S}} & \lesssim \delta+\left\|P_{h i} F(u)\right\|_{\mathcal{N}} \\
& \lesssim \delta+\left\|P_{h i}\left(\left|u_{l o}\right|^{\frac{4}{d-2 s_{c}}}\left|\nabla u_{l o}\right|\right)\right\|_{\mathcal{N}}+\left\|P_{h i}\left(\left|u_{l o}\right|^{\frac{4}{d-2 s_{c}}}\left|u_{h i}\right|\right)\right\|_{\mathcal{N}}+\left\|\left|u_{h i}\right|^{\frac{4}{d-2 s_{c}}}\left|u_{h i}\right|\right\|_{\mathcal{N}} .
\end{aligned}
$$

For the fourth term of (4.8), from Proposition 3.4 and (4.5) we have

$$
\left\|\left|u_{h i}\right|^{\frac{4}{d-2 s_{c}}}\left|u_{h i}\right|\right\|_{\mathcal{N}} \lesssim\left\||\nabla|^{s_{c}} u_{h i}\right\|_{L_{t}^{\infty} L_{x}^{2}}^{\frac{4}{d-2 s_{c}}}\left\|u_{h i}\right\|_{\delta} \lesssim \delta^{\frac{4}{d-2 s_{c}}}\left\|u_{h i}\right\|_{\delta}
$$

For the third term of (4.8), by Lemma 3.1, (3.12a) and (4.5), we have

$$
\begin{aligned}
& \left\|P_{h i}\left(\left|u_{l o}\right| \frac{4}{d-2 s_{c}}\left|u_{h i}\right|\right)\right\|_{\mathcal{N}} \\
\lesssim & \left\||\nabla|^{\frac{1-\varepsilon}{2}-s_{c}}\left(\left|u_{l o}\right| \frac{4}{d-2 s_{c}}\left|u_{h i}\right|\right)\right\|_{\mathcal{N}} \\
\lesssim & \left\||\nabla|^{\left(\frac{d}{2}-\frac{\left(d-2 s_{c}\right)\left(1+s_{c}+\varepsilon\right)}{4}\right)-} u_{l o}\right\|_{L_{t}^{\infty} L_{x}^{2}}^{\frac{4}{d-s_{c}}}\left\||\nabla|^{\left(-\frac{1-\varepsilon}{2}\right)+} u_{h i}\right\|_{\mathcal{S}} \\
\lesssim & \delta^{\frac{4}{d-2 s_{c}}}\left\|u_{h i}\right\|_{\mathcal{S}} .
\end{aligned}
$$

For the remained term of (4.8), by Lemma 3.1, (3.12a), (4.5) and (4.7a) we have

$$
\begin{aligned}
& \left\|P_{h i}\left(\left|u_{l o}\right|^{\frac{4}{d-2 s_{c}}}\left|\nabla u_{l o}\right|\right)\right\|_{\mathcal{N}} \\
\lesssim & \left\||x|^{\frac{1+\varepsilon}{2}}\left(\left|u_{l o}\right|^{\frac{4}{d-2 s_{c}}}\left|\nabla u_{l o}\right|\right)\right\|_{L_{t, x}^{2}} \\
\lesssim & \left\||\nabla|^{\left(-\frac{1-\varepsilon}{2}\right)+} \nabla u_{l o}\right\|\left\|_{\mathcal{S}}\right\||\nabla|^{\left(\frac{d}{2}-\frac{\left(d-2 s_{c}\right)\left(1+s_{c}+\varepsilon\right)}{4}\right)-} u_{l o} \|_{L_{t}^{\infty} L_{x}^{2}}^{\frac{4}{d-2 c_{c}^{2}}} \\
\lesssim & \eta^{\frac{1}{2}} \delta^{\frac{4}{d-2 s_{c}}} .
\end{aligned}
$$

Putting all these together, we obtain

$$
\left\|u_{h i}\right\|_{\mathcal{S}} \lesssim\left(\delta+\delta^{\frac{4}{d-2 s_{c}}}\right)\left(1+\left\|u_{h i}\right\|_{\mathcal{S}}\right),
$$

by Corollary 3.1, we know $\left\|u_{h i}\right\|_{\mathcal{S}}<\infty$, after reorganizing the term, we finally derive that

$$
\left\|u_{h i}\right\|_{\mathcal{S}\left(I \times \mathbb{R}^{d}\right)} \lesssim \delta+\delta^{\frac{4}{d-2 s_{c}}} .
$$

Thus, we complete the proof.

With the above preparation, we are now ready to prove Proposition 4.2. First we need the following particular form of Morawetz inequality which can be found in [31]. 
Lemma 4.3 (Morawetz inequality). Let $J$ be an interval, let $d \geq 3$ and let $\phi, G: J \times \mathbb{R}^{d} \rightarrow \mathbb{C}$ solve the equation

$$
i \partial_{t} \phi+\Delta \phi=F(\phi)+G .
$$

Let $\varepsilon>0$. If $\varepsilon$ is sufficiently small depending on $d$, then we have

$$
\begin{aligned}
& \int_{J} \int_{\mathbb{R}^{d}}\left(\frac{|\phi(t, x)|^{2}}{\langle x\rangle^{3+\varepsilon}}+\frac{|\phi|^{p+2}}{\langle x\rangle}+\frac{|\nabla \phi(t, x)|^{2}}{\langle x\rangle^{1+\varepsilon}}\right) d x d t \\
& \lesssim_{\varepsilon} \sup _{t \in J}\left\||\nabla|^{\frac{1}{2}} \phi(t, x)\right\|_{L_{x}^{2}}^{2} \\
& \quad+\int_{J} \int_{\mathbb{R}^{d}} G(t, x) \| \nabla \phi(t, x) \mid d x d t \\
& \quad+\int_{J} \int_{\mathbb{R}^{d}} \frac{1}{\langle x\rangle}|G(t, x) \| \phi(t, x)| d x t .
\end{aligned}
$$

Proof of Proposition 4.2. Let $P_{l_{0}}:=P_{<1}$, we substitute $\phi$ with $\phi=u_{l o}$, then the corresponding $G$ equals

$$
G:=P_{l o} F(u)-F\left(P_{l o} u\right) .
$$

Using Bernstein inequality and (4.5), we conclude that

$$
\int_{I} \int_{\mathbb{R}^{d}} \frac{\left|\nabla u_{l o}(t, x)\right|^{2}}{\langle x\rangle^{1+\varepsilon}} d x d t \lesssim_{\varepsilon} \delta+\int_{I} \int_{R^{d}}|G(t, x)|\left(\left|\nabla u_{l o}(t, x)\right|+\frac{\left|u_{l o}(t, x)\right|}{\langle x\rangle}\right) d x d t .
$$

Note that by Lemma 2.6

$$
\int_{I} \int_{\mathbb{R}^{d}} \frac{\left|\nabla u_{l o}(t, x)\right|^{2}}{|x|^{1+\varepsilon}} d x d t \lesssim \int_{I} \int_{\mathbb{R}^{d}} \frac{\left|\nabla u_{l o}(t, x)\right|^{2}}{\langle x\rangle^{1+\varepsilon}} d x d t
$$

it suffices to estimate

$$
\int_{I} \int_{R^{d}}|G(t, x)|\left(\left|\nabla u_{l o}(t, x)\right|+\frac{\left|u_{l o}(t, x)\right|}{\langle x\rangle}\right) d x d t \lesssim \delta^{c},
$$

where $c$ is a given constant to be chosen later. By the Hölder inequality and (4.7b), we estimate

$$
\begin{aligned}
& \int_{I} \int_{R^{d}}\left|G(t, x) \| \nabla u_{l o}(t, x)\right| d x d t \\
\lesssim & \|G\|_{L_{t}^{2} L_{x}^{\frac{2 d}{d+2\left(1-\varepsilon_{0}\right)}}\left\|\nabla u_{l o}\right\|} L_{L_{t}^{2} L_{x}^{\frac{2 d}{d-2\left(1-\varepsilon_{0}\right)}}} \\
\lesssim & \eta^{\frac{1}{2}}\|G\|_{L_{t}^{2} L_{x}^{\frac{2 d}{d+2\left(1-\varepsilon_{0}\right)}}} \cdot
\end{aligned}
$$


In dimension $d \geq 4$, by the Hölder inequality, (2.5) and (4.7b) we have

$$
\begin{aligned}
\int_{I} \int_{\mathbb{R}^{d}}|G(t, x)| \frac{\left|u_{l o}(t, x)\right|}{\langle x\rangle} d x d t & \lesssim\|G\|_{L_{t}^{2} L_{x}^{\frac{2 d}{d+2\left(1-\varepsilon_{0}\right)}}}\left\|\frac{u_{l o}}{\langle x\rangle}\right\|_{L_{t}^{2} L_{x}^{\frac{2 d}{d-2\left(1-\varepsilon_{0}\right)}}} \\
& \lesssim \eta^{\frac{1}{2}}\|G\|_{L_{t}^{2} L_{x}^{\frac{2 d}{d+2\left(1-\varepsilon_{0}\right)}}}
\end{aligned}
$$

Thus it is reduced to show

$$
\|G\|_{L_{t}^{2} L_{x}^{\frac{2 d}{d+2\left(1-\varepsilon_{0}\right)}}} \lesssim \eta \delta^{c} .
$$

We split $G$ into

$$
G:=P_{l o}\left[F(u)-F\left(u_{l o}\right)\right]-P_{h i}\left(F\left(u_{l o}\right)\right)
$$

We can show (4.15) via

$$
\begin{aligned}
& \quad\left\|P_{l o} \mathcal{O}\left(\left|u_{h i}\right|\left|u_{l o}\right|^{\frac{4}{d-2 s_{c}}}+\left|u_{h i}\right|^{1+\frac{4}{d-2 s_{c}}}\right)\right\|{ }_{L_{t}^{2} L_{x}^{\frac{2 d}{d+2\left(1-\varepsilon_{0}\right)}}}+\left\|P_{h i} F\left(u_{l o}\right)\right\|{ }_{L_{t}^{2} L_{x}^{\frac{2 d}{d+2\left(1-\varepsilon_{0}\right)}}} \\
& \lesssim\left\|P_{l o} \mathcal{O}\left(\left|u_{h i}\right|\left|u_{l o}\right|^{\frac{4}{d-2 s_{c}}}+\left|u_{h i}\right|^{1+\frac{4}{d-2 s_{c}}}\right)\right\|_{L_{t}^{2} L_{x}^{\frac{2 d}{d+2\left(1-\varepsilon_{0}\right)}}} \\
& +\left\|\nabla P_{h i} F\left(u_{l o}\right)\right\|_{L_{t}^{2} L_{x}^{\frac{2 d}{d+2\left(1-\varepsilon_{0}\right)}}} \cdot
\end{aligned}
$$

For (4.16), by (1.4), Sobolev embedding, Lemma 3.1, (4.7c), Bernstein, we estimate as

$$
\begin{aligned}
(4.16) & \lesssim\left\|\mathcal{O}\left(\left|u_{h i}\right|\left|u_{l o}\right|^{\frac{4}{d-2 s_{c}}}+\left|u_{h i}\right|^{1+\frac{4}{d-2 s_{c}}}\right)\right\|_{L_{t}^{2} L_{x}^{\frac{2 d}{d+2\left(1-\varepsilon_{0}\right)}}} \lesssim\left\||\nabla|^{s_{c}} \mathcal{u}\right\|_{L_{t}^{\infty} L_{x}^{2}}^{\frac{4}{d-2 s c}}\left\|u_{h i}\right\|_{L_{t}^{2} L_{x}^{\frac{2 d}{d-2}}} \\
& \lesssim \delta+\delta^{\frac{4}{d-2 s_{c}}} .
\end{aligned}
$$

Hence, it is remained to prove

$$
\left\|\left|\nabla u_{l o}\right|\left|u_{l o}\right|^{\frac{4}{d-2 s_{c}}}\right\|_{L_{t}^{2} L_{x}^{\frac{2 d}{d+2\left(1-\varepsilon_{0}\right)}}} \lesssim \delta^{c} .
$$

From (4.6) we have

$$
\left\||x|^{-\frac{1+\varepsilon}{2}} \nabla u_{l o}\right\|_{L_{t, x}^{2}} \lesssim \eta^{\frac{1}{2}},
$$

and by radial Sobolev embedding (2.5)

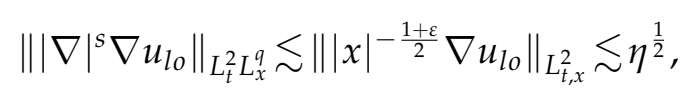


for some $q=\left(\frac{2(d-1)}{d-2-\varepsilon}\right)+, s=\left(\frac{d}{q}-\frac{d}{2}+\frac{1+\varepsilon}{2}\right)-$. By Bernstein we conclude that

$$
\left\|\nabla u_{l o}\right\|_{L_{t}^{2} L_{x}^{q}} \lesssim \eta^{\frac{1}{2}}
$$

By the Hölder inequality, we get

$$
\left\|\left|\nabla u_{l o}\right|\left|u_{l o}\right|^{\frac{4}{d-2 s_{c}}}\right\|_{L_{t}^{2} L_{x}^{\frac{2 d}{d+2\left(1-\varepsilon_{0}\right)}}} \lesssim\left\|\nabla u_{l o}\right\|_{L_{t}^{2} L_{x}^{q}}\left\|u_{l o}\right\|_{L_{t}^{\alpha} L_{x}^{\prime}}^{\frac{4}{d-2 s}}
$$

for some $r=\frac{2 d(d-1) p}{2\left(1-\varepsilon_{0}\right)(d-1)+d(1-\varepsilon)}->\frac{2 d}{d-2 s_{c}}$. By (4.5), we have

$$
\left\|u_{l o}\right\|_{L_{t}^{\infty} L_{x}^{r}} \lesssim \delta
$$

Combining the estimate for (4.16) and (4.17) we have

$$
\|G\|_{L_{t}^{2} \frac{2 d}{d+2\left(1-\varepsilon_{0}\right)}} \lesssim \eta+\delta^{\frac{4}{d-2 s_{c}}} .
$$

Now we can choose $c=\min \left\{1, \frac{4}{d-2 s_{c}}\right\}$ and $\delta(\eta)$ sufficiently small, then we complete the proof.

Remark 4.1. In order to use (2.5) in (4.14), we should ensure that $\frac{2 d}{d-2\left(1-\varepsilon_{0}\right)}<d$ which requires $d \geq 4$. For $d=3$, one can adapt the argument in [31] to bypass the obstacle, we omit the details here.

Corollary 4.1. Let $d \geq 4$, and $u: I \times \mathbb{R}^{d} \rightarrow \mathbb{C}$ be the spherically symmetric maximal-lifespan solution to (1.1) which obeys (1.4), (1.23) then

$$
\lim _{N \rightarrow \infty}\left[\left\|u_{\geq N}\right\|_{\mathcal{S}}+\frac{1}{N^{\frac{1+\varepsilon}{2}}}\left\||\nabla|^{-\frac{1-\varepsilon}{2}} \nabla u_{<N}\right\|_{\mathcal{S}}\right]=0
$$

In particular, for any $N>0$ being a dyadic integer, we have

$$
\left\|u_{\geq N}\right\|_{\mathcal{S}}+\frac{1}{N^{\frac{1+\varepsilon}{2}}}\left\||\nabla|^{-\frac{1-\varepsilon}{2}} \nabla u_{<N}\right\|_{\mathcal{S}}<\infty \quad \text { for all } N>0
$$

Proof. (4.22) comes from (4.7a), (4.7c) and the scaling invariance of the equation. Now we use (4.22) to prove (4.23). Since (4.22) implies (4.23) for $N$ is sufficiently large, it suffices to show that (4.23) also holds for $N$ is small. We may assume $N_{0}$ such that $N \geq N_{0}$

$$
\left\|u_{\geq N}\right\|_{\mathcal{S}}+\frac{1}{N^{\frac{1+\varepsilon}{2}}}\left\||\nabla|^{-\frac{1-\varepsilon}{2}} \nabla u_{<N}\right\|_{\mathcal{S}}<1 .
$$


For any $N<N_{0}$, we have

$$
\begin{aligned}
\left\|u_{>N}\right\|_{\mathcal{S}} & =\left\|u_{\geq N_{0}}\right\|_{\mathcal{S}}+\left\|u_{N<.<N_{0}}\right\|_{\mathcal{S}} \\
& \lesssim 1+\sum_{N<M<N_{0}}\left\|u_{M}\right\|_{\mathcal{S}} \\
& \lesssim 1+\sum_{N<M<N_{0}} M^{-\frac{1+\varepsilon}{2}}\left\||\nabla|^{\frac{1+\varepsilon}{2}} u_{M}\right\|_{\mathcal{S}} \\
& \lesssim 1+\sum_{N<M<N_{0}} 1 \\
& <\infty
\end{aligned}
$$

and

$$
\left\||\nabla|^{\frac{1+\varepsilon}{2}} u_{<N}\right\|_{\delta} \lesssim\left(\frac{N_{0}}{N}\right)^{\frac{1+\varepsilon}{2}} \frac{1}{N_{0}^{\frac{1+\varepsilon}{2}}}\left\||\nabla|^{\frac{1+\varepsilon}{2}} u_{<N_{0}}\right\|_{\delta}<\infty .
$$

Thus we complete the proof.

\section{The non-evacuation of energy}

In this part, we will prove that the energy can not evacuate from high frequency to low frequency by showing that $N(t)$ has a lower bound.

Proposition 5.1. Let $d \geq 4$, and let $u: I \times \mathbb{R}^{d} \rightarrow \mathbb{C}$ be the critical spherically symmetric maximal-lifespan solution to (1.1) which obeys (1.4), (1.23). Then

$$
\inf _{t \in I} N(t)>0 .
$$

Assume for contradiction that we have a critical solution $u: I \times \mathbb{R}^{d} \rightarrow \mathbb{C}$ obeying (1.4) and the hypothesis (1.23) but such that

$$
\inf _{t \in I} N(t)=0,
$$

we will obtain the following fact:

Lemma 5.1. Under the conditions of Proposition 5.1, we have

$$
\limsup _{N \rightarrow \infty} N^{\frac{3-\varepsilon}{2}-s_{c}}\left\|u_{\geq N}\right\|_{\mathcal{S}}<\infty .
$$

Proof. Let $\eta>0$ be a small number to be chosen later. By (4.22), there exists $\widetilde{N}_{0}>0$ such that

$$
\left\|u_{\geq \widetilde{N}_{0}}\right\|_{\mathcal{S}}+\frac{1}{\widetilde{N}_{0}^{\frac{1+\varepsilon}{2}}}\left\||\nabla|^{(1+\varepsilon) / 2} u_{<\widetilde{N}_{0}}\right\|_{\mathcal{S}} \lesssim \eta
$$


By scaling invariance, we may assume $\widetilde{N}_{0}=1$, thus

$$
\begin{aligned}
& \left\||\nabla|^{(1+\varepsilon) / 2} u_{<1}\right\|_{\mathcal{S}} \leq \eta \\
& \left\|u_{\geq 1}\right\|_{\mathcal{S}} \leq \eta
\end{aligned}
$$

We claim that:

Claim 5.1. For any given $\delta>0$ such that

$$
\left\|u_{\geq N}\right\|_{\delta} \leq \eta N^{-(3-\varepsilon) / 2+s_{c}}+\delta \text { for all } N \geq 1,
$$

then

$$
\left\|u_{\geq N}\right\|_{\mathcal{S}} \leq \eta N^{-(3-\varepsilon) / 2+s_{c}}+\frac{\delta}{2} \text { for all } N \geq 1
$$

Assuming the claim, by iterating the above procedure, we will conclude that

$$
\left\|u_{\geq N}\right\|_{\mathcal{S}} \leq \eta N^{-\frac{3-\varepsilon}{2}+s_{c}} \quad \text { for all } N \geq 1 .
$$

Now we are dedicated to proving the claim. Indeed, by choosing $0<\delta \leq \eta$ such that (5.5) holds. Furthermore, we can take a dyadic number $N_{0} \geq 1$ such that $\eta N_{0}^{-\frac{3-\varepsilon}{2}+s_{c}} \sim \delta$, then

$$
\left\|u_{\geq N}\right\|_{\delta} \lesssim \eta N^{-\frac{3-\varepsilon}{2}+s_{c}} \quad \text { for all } 1 \leq N \leq N_{0}
$$

and

$$
\left\|u_{\geq N_{0}}\right\|_{\delta} \lesssim \delta
$$

Let $N \geq 1$, applying $P_{\geq N}$ to both sides of (1.1) we have

$$
\left(i \partial_{t}+\Delta\right) u_{\geq N}=P_{\geq N} F(u) .
$$

Hence, by weighted Strichartz estimate (3.2) we have

$$
\left\|u_{\geq N}\right\|_{\mathcal{S}} \lesssim\left\||\nabla|^{s_{c}} u_{\geq N}\left(t_{0}\right)\right\|_{L_{x}^{2}}+\left\|P_{\geq N} F(u)\right\|_{\mathcal{N}},
$$

for any $t_{0} \in I$. As $\inf _{t \in I} N(t)=0$, we have

$$
\inf _{t_{0} \in I}\left\||\nabla|{ }^{s_{c}} u_{\geq N}\left(t_{0}\right)\right\|_{L_{x}^{2}}=0 .
$$

Thus

$$
\left\|u_{\geq N}\right\|_{\delta} \lesssim\left\|P_{\geq N} F(u)\right\|_{\mathcal{N}} .
$$


We split $F(u)$ as

$$
\begin{aligned}
F(u)= & F\left(u_{<N_{0}}\right)+\mathcal{O}\left(\left|u_{\geq N_{0}}\right|\left(\left|u_{<1}\right|^{\frac{4}{d-2 s_{c}}}+\left|P_{<1} u_{\leq N_{0}}\right|^{\frac{4}{\left.\right|^{-2 s_{c}}}}\right)\right) \\
& +\mathcal{O}\left(\left|u_{\geq N_{0}}\right|\left(\left|u_{\geq 1}\right|^{\frac{4}{d-2 s_{c}}}+\left|P_{\geq 1} u_{\leq N_{0}}\right|^{\frac{4}{d-2 s_{c}}}\right)\right) .
\end{aligned}
$$

So that we have

$$
\begin{aligned}
\text { RHS of }(5.12) \lesssim & \left\|P_{\geq N} \mathcal{O}\left(\left|u_{\geq N_{0}}\right|\left(\left|u_{\geq 1}\right|+\left|P_{\geq 1} u_{\leq N_{0}}\right|\right)^{\frac{4}{d-2 s_{c}}}\right)\right\|_{\mathcal{N}} \\
& +\left\|P_{\geq N} \mathcal{O}\left(\left|u_{\geq N_{0}}\right|\left(\left|u_{<1}\right|+\left|P_{<1} u_{\leq N_{0}}\right|\right)^{\frac{4}{d-2 s_{c}}}\right)\right\|_{\mathcal{N}} \\
& +\left\|P_{\geq N} F\left(u_{<N_{0}}\right)\right\|_{\mathcal{N}} .
\end{aligned}
$$

By (3.4), (5.4b), (5.8), we have

$$
\begin{aligned}
& \left\|P_{\geq N} \mathcal{O}\left(\left|u_{\geq N_{0}}\right|\left|u_{\geq 1}\right|^{\frac{4}{d-2 s_{c}}}\right)\right\|_{\mathcal{N}} \\
\lesssim & \left\|\left|u_{\geq N_{0}}\right|\left|u_{\geq 1}\right|^{\frac{4}{d-2 s_{c}}}\right\|_{\mathcal{N}} \\
\lesssim & \left\||\nabla|^{s_{C}} u_{\geq 1}\right\|_{L_{t}^{\infty} L_{x}^{2}}^{\frac{4}{d-2 s_{c}}}\left\|u_{\geq N_{0}}\right\|_{\mathcal{S}} \\
\lesssim & \eta^{\frac{4}{d-2 s_{c}}} \delta .
\end{aligned}
$$

The other term in (5.13) is estimated similarly.

For (5.14), by Lemma 3.1, (3.12a) (5.4a) and (5.8) we obtain

$$
\begin{aligned}
& \left\|P_{\geq N} \mathcal{O}\left(\left|u_{\geq N_{0}}\right|\left|u_{<1}\right|^{\frac{4}{d-2 s_{c}}}\right)\right\|_{\mathcal{N}} \\
\lesssim & \left\||\nabla|^{\frac{1-\varepsilon}{2}-s_{c}} \mathcal{O}\left(\left|u_{\geq N_{0}}\right|\left|u_{<1}\right|^{\frac{4}{d-2 s_{c}}}\right)\right\|_{\mathcal{N}} \\
\lesssim & \left\||\nabla|^{\left(\frac{d}{2}-\frac{\left(d-2 s_{c}\right)\left(1+s_{c}+\varepsilon\right)}{4}\right)-} u_{<1}\right\|_{L_{t}^{\infty} L_{x}^{2}}^{\frac{4}{d-2 s_{c}}}\left\||\nabla|^{\left(-\frac{1-\varepsilon}{2}\right)+} u_{\geq N_{0}}\right\|_{\mathcal{S}} \\
\lesssim & \eta{ }^{\frac{4}{d-2 s_{c}}} \delta .
\end{aligned}
$$

The other term of (5.14) is estimated similarly.

For the (5.15), by Lemma 3.1 and (3.12b)

$$
\begin{aligned}
\left\|P_{\geq N} F\left(u_{<N_{0}}\right)\right\|_{\mathcal{N}} & \lesssim N^{-\frac{3-\varepsilon}{2}+s_{c}}\left\||x|^{\frac{1+\varepsilon}{2}} \mathcal{O}\left(\left|u_{<N_{0}}^{p} \nabla u_{<N_{0}}\right|\right)\right\|_{L_{t, x}^{2}} \\
& \lesssim N^{-\frac{3-\varepsilon}{2}+s_{c}}\left\||\nabla|^{\frac{1+\varepsilon}{2}} u_{<N_{0}}\right\|_{L_{t}^{\infty} L_{x}^{2}}^{p}\left\||\nabla|^{\frac{1-\varepsilon}{2}-s_{c}+p\left(s_{c}-\frac{1+\varepsilon}{2}\right)} \nabla u_{<N_{0}}\right\|_{\mathcal{S}} .
\end{aligned}
$$

Since by (5.4a) and (5.7) we have

$$
\begin{aligned}
\left\||\nabla|^{\frac{1+\varepsilon}{2}} u_{<N_{0}}\right\|_{L_{t}^{\infty} L_{x}^{2}} & \lesssim\left\||\nabla|^{\frac{1+\varepsilon}{2}} u_{\leq 1}\right\|_{L_{t}^{\infty} L_{x}^{2}}+\sum_{1<M<N_{0}}\left\||\nabla|^{\frac{1+\varepsilon}{2}} u_{M}\right\|_{L_{t}^{\infty} L_{x}^{2}} \\
& \lesssim \eta+\eta \sum_{1<M<N_{0}} M^{\frac{1+\varepsilon}{2}} M^{-\frac{3-\varepsilon}{2}} \\
& \lesssim \eta
\end{aligned}
$$


and

$$
\begin{aligned}
& \left\||\nabla|^{\frac{1-\varepsilon}{2}-s_{c}+p\left(s_{c}-\frac{1+\varepsilon}{2}\right)} \nabla u_{<N_{0}}\right\|_{\mathcal{S}} \\
\lesssim & \left\||\nabla|^{\frac{1-\varepsilon}{2}-s_{c}+p\left(s_{c}-\frac{1+\varepsilon}{2}\right)} \nabla u_{<1}\right\|_{\mathcal{S}}+\sum_{1<M<N_{0}}\left\||\nabla|^{\frac{1-\varepsilon}{2}-s_{c}+p\left(s_{c}-\frac{1+\varepsilon}{2}\right)} \nabla u_{M}\right\|_{\mathcal{S}} \\
\lesssim & \eta+\eta \sum_{1<M<N_{0}} M^{\frac{1-\varepsilon}{2}-s_{c}+p\left(s_{c}-\frac{1+\varepsilon}{2}\right)} M M^{-\frac{3-\varepsilon}{2}+s_{c}} \\
\lesssim \eta &
\end{aligned}
$$

Thus

$$
\left\|P_{\geq N} F\left(u_{<N_{0}}\right)\right\|_{\mathcal{N}} \lesssim \eta^{p} \eta N^{-\frac{3-\varepsilon}{2}+s_{c}} .
$$

Combining the separated parts contributed to $\left\|u_{>N}\right\|_{\mathcal{S}}$, we have

$$
\left\|u_{>N}\right\|_{\delta} \lesssim \eta^{p}\left(\eta N^{-\frac{3-\varepsilon}{2}+s_{c}}+\delta\right) \text { for } N \geq 1 .
$$

By choosing $\eta$ sufficiently small, we complete the proof.

Proof of Proposition 5.1. Now we can illuminate that $\inf _{t \in I} N(t)=0$ is incompatible with energy-conservation. In fact, by (5.2), for sufficiently large $N$, we have

$$
\left\|\nabla P_{N} u\right\|_{L_{t}^{\infty} L_{x}^{2}} \lesssim N^{-\frac{1-\varepsilon}{2}}
$$

and for each dyadic number $N$

$$
\left\|\nabla P_{N} u\right\|_{L_{t}^{\infty} L_{x}^{2}} \lesssim N^{1-s_{c}}
$$

Thus, by choosing $M$ sufficiently large

$$
\|\nabla u\|_{L_{t}^{\infty} L_{x}^{2}} \lesssim\left\|\nabla u_{<M^{-1}}\right\|_{L_{t}^{\infty} L_{x}^{2}}+\left\|\nabla u_{M^{-1} \leq .<M}\right\|_{L_{t}^{\infty} L_{x}^{2}}+\left\|\nabla u_{\geq M}\right\|_{L_{t}^{\infty} L_{x}^{2}}
$$

as $\inf _{t \in I} N(t)=0$, we may choose a time sequence $\left\{t_{i}\right\} \in I$ such that $N\left(t_{i}\right) \rightarrow 0$, and by dominated convergence theorem we conclude that

$$
\left\|\nabla u\left(t_{i}\right)\right\|_{L_{x}^{2}} \rightarrow 0 \quad \text { as } N\left(t_{i}\right) \rightarrow 0 .
$$

By interpolation

$$
\|u\|_{L_{x}^{p+2}} \lesssim\|u\|_{\frac{d p}{2}}^{\theta}\|u\|_{\frac{2 d}{d-2}}^{1-\theta} \lesssim\left\||\nabla|^{s^{c}} u\right\|_{L_{x}^{2}}^{\theta}\|\nabla u\|_{L_{x}^{2}}^{1-\theta} \rightarrow 0 \quad \text { as } N\left(t_{i}\right) \rightarrow 0,
$$

where $0<\theta<1$.Thus

$$
E(u)=\int \frac{1}{2}|\nabla u|^{2}+\frac{1}{p+2}|u|^{p+2} d x \rightarrow 0 \quad \text { as } N\left(t_{i}\right) \rightarrow 0 .
$$

By the energy conservation law of (1.1), (5.21) implies that $u \equiv 0$, which is impossible. 


\section{Rule out the critical solution}

Theorem 6.1. Let $d \geq 4$, and let $u: I \times \mathbb{R}^{d} \rightarrow \mathbb{C}$ be the critical maximal-lifespan spherically symmetric solution to (1.1) which obeys (1.4), (1.23). Suppose that $u$ is not identically zero, then I is bounded.

Proof. By (1.13) and the fact that $N(t)$ has lower bound, we may choose $N$ sufficiently small such that

$$
\left\||\nabla|{ }^{s_{c}} u_{>N}\right\|_{L_{x}^{\frac{2 d}{d-2}}} \gtrsim 1
$$

then integrating with respect to the time variable over the interval $I$, we have

$$
|I|^{\frac{1}{2}} \lesssim\left\||\nabla|^{S^{c}} u_{>N}\right\|_{L_{t}^{2} L_{x}^{\frac{2 d}{d-2}}}
$$

By (4.23) and (3.3), we know $\left\||\nabla|^{s^{c}} u_{>N}\right\|_{L_{t}^{2} L_{x}^{\frac{2 d}{d-2}}}<\infty$, which implies $|I|<\infty$.

Theorem 6.1 means that $u$ blows up in finite time, thus by Corollary 1.1, $N(t)$ does not have upper bound in $I$, which is inconsistent with (1.23).

\section{Appendix}

In this part, we dedicate to proving Lemma 1.1. First we recall the definition of Strichartz norm and Strichartz estimate.

Definition 6.1 (Admissible pair). Let $d \geq 4$, we call a pair of exponent $(q, r)$ admissible if

$$
\frac{2}{q}=d\left(\frac{1}{2}-\frac{1}{r}\right) \quad \text { with } 2 \leq q \leq \infty .
$$

For a time interval $I$, we define Strichartz norm $S(I)$ as

$$
\|u\|_{\mathrm{S}(\mathrm{I})}:=\sup \left\{\|u\|_{L_{t}^{q} L_{x}^{r}\left(I \times \mathbb{R}^{d}\right)}:(q, r) \text { admissible }\right\} .
$$

We also define the dual of $\mathrm{S}(\mathrm{I})$ by $\mathrm{N}(\mathrm{I})$, we note that

$$
\|u\|_{\mathrm{N}(\mathrm{I})} \lesssim\|u\|_{L_{t}^{q^{\prime}} L_{x}^{r^{\prime}\left(I \times \mathbb{R}^{d}\right)}} \text { for any admissible pair }(q, r) .
$$

Proposition 6.1 (Strichartz estimate). Let $u: I \times \mathbb{R}^{d} \rightarrow \mathbb{C}$ be a solution to

$$
\left(i \partial_{t}+\Delta\right) u=F
$$

and let $s \geq 0$, then

$$
\left\||\nabla|^{s} u\right\|_{S(I)} \lesssim\left\|u\left(t_{0}\right)\right\|_{\dot{H}_{x}^{s}}+\left\||\nabla|^{s} F\right\|_{N(I)},
$$

for any $t_{0} \in I$. 
In the proof of Lemma 1.1, we need the following result. One can carry over the proof of Lemma 3.4 in [35] verbatim.

Lemma 6.1 (Persistence of regularity). Let I be a compact time interval, and $u$ be a solution to (1.10) obeying

$$
\|u\|_{L_{t, x}{ }^{2}}{ }_{\left(I \times \mathbb{R}^{d}\right)} \leq M, \quad\left\||\nabla|^{s_{c}} e\right\|_{\mathrm{N}(I)} \leq L \ll 1,
$$

then we have

$$
\left\||\nabla|{ }^{s_{c}} u\right\|_{\mathrm{S}(\mathrm{I})} \leq C(M)\left\|u_{0}\right\|_{\dot{H}_{x}^{s_{c}}} .
$$

In what follows, we denote

$$
\begin{aligned}
& X(I):=L_{t}^{\frac{2\left(d-2 s_{c}+2\right)}{\left(d-2 s_{c}\right)\left(1-s_{c}\right)}} L_{x}^{\frac{2\left(d-2 s_{c}+2\right)}{d-2 s_{c}}}\left(I \times \mathbb{R}^{d}\right), \\
& Y(I):=L_{t}^{\frac{2\left(d-2 s_{c}+2\right)}{\left(d+4-2 s_{c}\right)\left(1-s_{c}\right)}} L_{x}^{\frac{2\left(d-2 s_{c}+2\right)}{d-2 s_{c}+4}}\left(I \times \mathbb{R}^{d}\right), \\
& X^{\prime}(I):=L_{t}^{\frac{2\left(d-2 s_{c}+2\right)}{\left(d-2 s_{c}\right)\left(1-s_{c}\right)}} \dot{H}^{s_{c}, \frac{2 d\left(d-2 s_{c}+2\right)}{d^{2}+4 s_{c}-4 s_{c}^{2}}}\left(I \times \mathbb{R}^{d}\right), \\
& Y^{\prime}(I):=L_{t}^{\frac{2\left(d-2 s_{c}+2\right)}{\left(d+4-2 s_{c}\right)\left(1-s_{c}\right)}} \dot{H}_{x}^{s_{c}, \frac{2 d\left(d-2 s_{c}+2\right)}{d^{2}+4 d-4 s_{c}^{2}+4 s_{c}}}\left(I \times \mathbb{R}^{d}\right) .
\end{aligned}
$$

Obviously, we have $X^{\prime}(I) \hookrightarrow X(I), Y^{\prime}(I) \hookrightarrow Y(I)$.

Remark 6.1. The reason we choose the particular form of $X(I)$ and $Y(I)$ stems from the following fact: by dispersive estimate and Hardy-Littlewood-Sobolev inequality we can obtain relatively neat nonlinear estimate

$$
\left\|\int_{0}^{t} e^{i t \Delta}|u|^{p} u(s) d s\right\|_{X(I)} \lesssim\left\||u|^{p} u\right\|_{Y(I)} \lesssim\|u\|_{X(I)}^{p+1} .
$$

Next we will present some nonlinear estimates.

Lemma 6.2. Let $F(u)=|u|^{p} u$ for some $p>0$ and let $0<s<1$. For $1<r, r_{1}, r_{2}, \infty$ such that $\frac{1}{r}=\frac{1}{r_{1}}+\frac{p}{r_{2}}$, we have

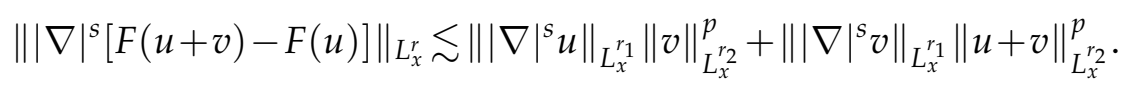

Lemma 6.3. Let $d \geq 4$, then with spacetime norms over $I \times \mathbb{R}^{d}$, we have

$$
\begin{aligned}
& \|F(u)\|_{Y(I)} \lesssim\|u\|_{X(I)^{\prime}}^{1+p} \\
& \left\||\nabla|^{s_{c}}[F(u)-F(v)]\right\|_{L_{t}^{2} L_{x}^{\frac{2 d}{d+2}}} \lesssim\|u-v\|_{X(I)}^{\frac{4}{d-2 s_{c}}}\|v\|_{\dot{S}^{s_{c}}}+\|u\|_{X(I)}^{\frac{4}{d-2 s_{c}}}\|u-v\|_{\dot{S}^{s_{c}}(I)} .
\end{aligned}
$$


Proof. (6.11a) comes directly from the definition of $X(I)$ and $Y(I)$. (6.11b) from Lemma 6.2 .

In order to prove Lemma 1.1, we primarily establish the short-time perturbation result.

Lemma 6.4 (Short-time perturbation). Let $d \geq 4$, I be a compact interval, $\tilde{u}: I \times \mathbb{R}^{d} \rightarrow \mathbb{C}$ be solution to the equation

$$
\left\{\begin{array}{l}
\left(i \partial_{t}+\Delta\right) \tilde{u}=F(\tilde{u})+e \\
\tilde{u}(0)=\tilde{u}_{0} \in \dot{H}^{s_{c}}
\end{array}\right.
$$

Suppose

$$
\|\tilde{u}\|_{L_{t}^{\infty} \dot{H}^{s_{c}}\left(I \times \mathbb{R}^{d}\right)} \leq E .
$$

Let $0 \in I$ and $u_{0} \in \dot{H}_{x}^{s_{c}}\left(\mathbb{R}^{d}\right)$. Then there exits $\varepsilon_{0}, \delta>0$ (depending on $E$ ) with the following properties hold: $0<\varepsilon<\varepsilon_{0}$, if

$$
\|\tilde{u}\|_{X(I)} \leq \delta, \quad\left\|u_{0}-\tilde{u}_{0}\right\|_{\dot{H}^{s c}}+\|e\|_{Y^{\prime}(I)} \leq \varepsilon,
$$

then there exits $u: I \times \mathbb{R}^{d} \rightarrow \mathbb{C}$ solving

$$
\left(i \partial_{t}+\Delta\right) u=|u|^{p} u \quad \text { with } u(0)=u_{0},
$$

satisfying

$$
\begin{aligned}
& \left\||\nabla|^{s_{c}}(u-\tilde{u})\right\|_{S(I)} \lesssim \varepsilon^{c}, \\
& \left\||\nabla|^{s_{C}} u\right\|_{S(I)} \lesssim E, \\
& \left\||\nabla|^{s_{c}}\left(|u|^{p} u-|\tilde{u}| \tilde{u}\right)\right\|_{N(I)} \lesssim \varepsilon^{c},
\end{aligned}
$$

where $c>0$ is a given constant.

Proof. First, we show that $\|u\|_{X(I)} \lesssim \delta$. Indeed by Duhamel formula (1.3)

$$
\begin{aligned}
\left\|e^{i t \Delta} \tilde{u}_{0}\right\|_{X(I)} & \lesssim\|\tilde{u}\|_{X(I)}+\|F(\tilde{u})\|_{Y(I)}+\|e\|_{Y^{\prime}(I)} \\
& \lesssim \delta+\delta^{1+\frac{4}{d-2 s_{c}}+\varepsilon .}
\end{aligned}
$$

By (6.12) and triangle inequality we have

$$
\left\|e^{i t \Delta} u_{0}\right\|_{X(I)} \lesssim \delta
$$

Then using Strichartz estimate (6.9) and (6.11a), we have

$$
\|u\|_{X(I)} \lesssim \delta+\|F(u)\|_{Y(I)} \lesssim \delta+\|u\|_{X(I)}^{1+\frac{4}{d-2 s_{c}}} .
$$


By continuity argument we have $\|u\|_{X(I)} \lesssim \delta$.

We let $w=u-\tilde{u}$, thus $w$ satisfies

$$
\left(i \partial_{t}+\Delta\right) w=F(u)-F(\tilde{u})-e, \quad w(0)=u_{0}-\tilde{u}_{0} .
$$

By Strichartz estimate (6.9) we have

$$
\begin{aligned}
\|w\|_{X(I)} & \lesssim\left\|e^{i t \Delta} w(0)\right\|_{X(I)}+\|e\|_{Y(I)}+\|F(u)-F(v)\|_{Y(I)} \\
& \lesssim \varepsilon+\left\{\|\tilde{u}\|_{X(I)}^{\frac{4}{d-2 s_{c}}}+\|u\|_{X(I)}^{\frac{4}{d-2 s_{c}}}\right\}\|w\|_{X(I)} \\
& \lesssim \varepsilon+\delta \frac{4}{d-2 s_{c}}\|w\|_{X(I)} .
\end{aligned}
$$

Thus by choosing $\delta$ sufficiently small, we have

$$
\|w\|_{X(I)} \lesssim \varepsilon
$$

By Strichartz estimate and (6.12) and (6.11b), we have

$$
\begin{aligned}
\left\||\nabla|^{s_{c}} w\right\|_{S(I)} & \lesssim\left\|u_{0}-\tilde{u}_{0}\right\|_{\dot{H}^{s_{c}}}+\|e\|_{Y^{\prime}(I)}+\left\||\nabla|^{s_{c}}[F(u)-F(\tilde{u})]\right\|_{L_{t, x} \frac{2(d+2)}{d+4}} \\
& \lesssim \mathcal{E}+\left\||\nabla|^{s_{c}} \tilde{\mathcal{u}}\right\|_{S(I)}\|w\|_{X(I)}^{\frac{4}{d-2 s_{c}}}+\left\||\nabla|^{s_{c}} w\right\|_{S(I)}\|u\|_{X(I)}^{\frac{4-2 s_{c}}{d+(I)}}
\end{aligned}
$$

By (6.12) and the persistence of regularity results, we have $\left\||\nabla|^{s_{c}} \tilde{u}\right\|_{S(I)} \leq C(\delta) E$. For (6.13b), by (6.13a) and Strichartz estimate we have

$$
\begin{aligned}
& \left\||\nabla|^{s^{c}} u\right\|_{S(I)} \\
\lesssim & \mathcal{E}^{c}+\left\||\nabla|^{s_{c}} \tilde{u}_{0}\right\|_{L_{x}^{2}}+\left\|\left(|\tilde{u}|^{p} \tilde{u}\right)\right\|_{Y^{\prime}(I)}+\|e\|_{Y^{\prime}(I)} \\
\lesssim & \mathcal{E}^{c}+E+\|\tilde{u}\|_{X(I)}^{\frac{4}{d-2 s_{c}}}\|\tilde{u}\|_{X^{\prime}(I)} \\
\lesssim & \mathcal{E}^{c}+E+C(\delta) E \\
\lesssim E &
\end{aligned}
$$

Now (6.13c) can be deduced from Lemma 6.2 and (6.13a).

Proof of Lemma 1.1. First note that $\|\tilde{u}\|_{L_{t, x} \frac{(d+2) p}{2}\left(I \times \mathbb{R}^{d}\right)} \leq L$, by the persistence of regularity, we have $\|\tilde{u}\|_{X(I)} \lesssim C(E, L)$. Then we may subdivide $I$ into (finitely many, depending on $\delta$ and $L)$ intervals $J_{k}=\left[t_{k}, t_{k+1}\right)$ so that

$$
\|\tilde{u}\|_{X(I)} \sim \delta,
$$

then we can use the short-time perturbation results and bootstrap argument to obtain Lemma 1.1. 


\section{Acknowledgements}

This work was supported in part by the National Natural Science Foundation of China under grant No. 11671047 and No. 11726005. Yang Jianwei-Urbain is supported by the LabEx MME-DII.

\section{References}

[1] J. Bourgain, Global wellposedness of defocusing critical nonlinear Schrödinger equation in the radial case, J. Amer. Math. Soc., 12 (1999), 145-171.

[2] T. Cazenave, Semilinear schrödinger equations, American Mathematical Soc., 2003.

[3] T. Cazenave and F. B. Weissler, The Cauchy problem for the nonlinear Schrodinger equations in $H^{s}$, Non-Linear Anal., 14(10) (1990), 807-836.

[4] J. Colliander, M. Keel, G. Staffilani, H. Takaoka, and T. Tao, Global existence and scattering for rough solutions of a nonlinear Schrödinger equation on $\mathbb{R}^{3}$, Commun. Pure Appl. Math., 57(8) (2003), 987-1014.

[5] J. Colliander, M. Keel, G. Staffilani, H. Takaoka, and T. Tao, Global well-posedness and scattering for the energy-critical nonlinear Schrödinger equation in $\mathbb{R}^{3}$, Ann. Math., 167 (2008), 767-865.

[6] B. Dodson, Global well-posedness and scattering for the defocusing, $L^{2}$-critical, nonlinear Schrödinger equation when $d \geq 3$, J. Amer. Math. Soc., 25 (2012), 429-463.

[7] B. Dodson, Global well-posedness and scattering for the defocusing, $L^{2}$-critical, nonlinear Schrödinger equation when $d=2$, Duke Math. J., 165 (2016), 3435-3516.

[8] B. Dodson, Global well-posedness and scattering for the defocusing, $L^{2}$-critical, nonlinear Schrödinger equation when $d=1$, Amer. J. Math., 138 (2016), 531-569.

[9] B. Dodson, Global well-posedness and scattering for the mass critical nonlinear Schrödinger equation with mass below the mass of the ground state, Adv. Math., 285 (2015), 1589-1618.

[10] B. Dodson, Global well-posedness and scattering for the focusing, energy-critical nonlinear Schrödinger problem in dimension $d=4$ for initial data below a ground state threshold, arXiv preprint arXiv:1409.1950, (2014).

[11] B. Dodson, C. Miao, J. Murphy, and J. Zheng, The defocusing quintic NLS in four space dimensions, Annales de l'Institut Henri Poincare/Analyse non lineaire, 34 (2017), 759-787.

[12] J. Holmer and S. Roudenko, A sharp condition for scattering of the radial 3D cubic nonlinear Schrödinger equation, Commun. Math. Phys., 282(2) (2008), 435-467.

[13] M. Grillakis, On nonlinear Schrödinger equations, Commun. PDE, 25 (2000), 1827-1844.

[14] C. E. Kenig and F. Merle, Scattering for $\dot{H}^{1 / 2}$ bounded solutions to the cubic, defocusing NLS in 3 dimensions, Trans. Amer. Math. Soc., 362 (2010), 1937-1962.

[15] C. E. Kenig and F. Merle, Global well-posedness, scattering and blow-up for the energycritical, focusing, non-linear Schrödinger equation in the radial case, Invent. Math., 166 (2006), 645-675.

[16] C. E. Kenig and F. Merle, Scattering for $\dot{H}^{1 / 2}$ bounded solutions to the cubic, defocusing NLS in 3 dimensions, Trans. Amer. Math. Soc., 362 (2010), 1937-1962.

[17] R. Killip and M. Visan, The focusing energy-critical nonlinear Schrödinger equation in dimensions five and higher, Amer. J. Math., 132 (2010), 361-424.

[18] R. Killip and M. Visan, Energy-supercritical NLS: critical $\dot{H}^{s}$-bounds imply scattering, Commun. PDE, 35 (2010), 945-987. 
[19] R. Killip and M. Visan, Nonlinear Schrödinger equations at critical regularity, Evolution Equations, 325-437, Clay Math. Proc., 17, Amer. Math. Soc., Providence, RI, 2013.

[20] R.Killip and M. Visan, Global well-posedness and scattering for the defocusing quintic NLS in three dimensions, Anal. Partial Differential Equations, 5(4) (2011), 855-885.

[21] R. Killip, T. Tao, and M. Visan, The cubic nonlinear Schrödinger equation in two dimensions with radial data, J. Euro. Math. Soc., (JEMS) 11 (2009), 1203-1258.

[22] R. Killip, M. Visan and X. Zhang, The mass-critical nonlinear Schrödinger equation with radial data in dimensions three and higher, Anal. PDE, 1 (2008), 229-266.

[23] C. Lu and J. Zheng, The radial defocusing energy-supercritical NLS in dimension four, J. Differential Equations, 262(8) (2017), 4390-4414.

[24] C. Miao, J. Murphy, and J. Zheng, The defocusing energy-supercritical NLS in four space dimensions, J. Funct. Anal., 267(6) (2014), 1662-1724.

[25] J. Murphy, Inter-critical NLS: critical $\dot{H}^{s}$-bounds imply scattering, SIAM J. Math. Anal., 46 (2014), 939-997.

[26] J. Murphy, The defocusing $\dot{H}^{1 / 2}$-critical NLS in high dimensions, Discrete Contin. Dyn. Syst. Series A, 34 (2014), 733-748.

[27] J. Murphy, The radial defocusing nonlinear Schrödinger equation in three space dimensions, Commun. Partial Differential Equations, 40 (2015), 265-308.

[28] E. Ryckman and M. Visan, Global well-posedness and scattering for the defocusing energycritical nonlinear Schrödinger equation in $R^{1+4}$, Amer. J. Math., 129 (2007), 1-60.

[29] E. M. Stein, Harmonic Analysis : Real-Variable Methods, Orthogonality, and Oscillatory Integrals, Princeton University Press, 2006.

[30] T. Tao, Global well-posedness and scattering for the higher-dimensional energy-critical nonlinear Schrödinger equation for radial data, New York J. Math., 11 (2005), 57-80.

[31] T. Tao, M. Visan, and X. Zhang, Global well-posedness and scattering for the defocusing mass-critical nonlinear Schrödinger equation for radial data in high dimensions, Duke Math. J., 140 (2007), 165-202.

[32] T. Tao, M. Visan, and X. Zhang, Minimal-mass blowup solutions of the mass-critical NLS, Forum Math., 20 (2008), 881-919.

[33] M. C. Vilela, Regularity of solutions to the free Schrödinger equation with radial initial data, Illinois J. Math., 45 (2001), 361-370.

[34] M. Visan, The defocusing energy-critical nonlinear Schrödinger equation in higher dimensions, Duke Math. J., 138 (2007), 281-374.

[35] M. Visan, The Defocusing Energy-Critical Nonlinear Schrödinger Equation in Dimensions Five and Higher, Ph.D. Thesis, UCLA, 2006.

[36] M. Visan, Global well-posedness and scattering for the defocusing cubic nonlinear Schrödinger equation in four dimensions, Int. Math. Research Notices, 5 (2012), 1037-1067. 